RE|BS

Beiträge zur Immobilienwirtschaft $\mathbf{\square}$

\title{
MIETSPIEGEL AUS ÖKONOMISCHER SICHT - VORSCHLÄGE FÜR EINE NEUREGULIERUNG
}

Dr. Oliver Lerbs

Zentrum für Europäische Wirtschaftsforschung (ZEW)

Prof. Dr. Steffen Sebastian

IREBS, Universität Regensburg und ZEW 
Herausgeber: IRE|BS International Real Estate Business School, Universität Regensburg www.irebs.de

ISSN 2197-7720

Copyright @ IRE|BS International Real Estate Business School 2015, alle Rechte vorbehalten

Verantwortlich für den Inhalt dieses Bandes: Prof. Dr. Steffen Sebastian, Lehrstuhl für Immobilienfinanzierung, Universität Regensburg

\section{RECHTLICHE HINWEISE}

\section{ZUGANG}

Die Publikation von und der Zugang zu Informationen in dieser Studie kann durch lokale Vorschriften in gewissen Ländern eingeschränkt sein. Diese Studie richtet sich ausdrücklich nicht an Personen in Staaten, in denen (aufgrund der Staatsangehörigkeit bzw. des Wohnsitzes der jeweiligen Person oder aus anderen Gründen) entsprechende Einschränkungen gelten. Insbesondere richtet sich die Studie nicht an Bürger der USA sowie an Personen, die in den USA oder in einem ihrer Territorien, Besitzungen oder sonstigen Gebieten, die der Gerichtshoheit der USA unterstehen, wohnhaft sind oder dort ihren gewöhnlichen Aufenthalt haben. Personen, für welche entsprechende Beschränkungen gelten, dürfen nicht, weder online noch in anderer Form, auf diese Studie zugreifen.

\section{KEIN ANGEBOT}

Der Inhalt dieser Studie dient ausschließlich Informationszwecken und stellt keine Werbung, kein Angebot und keine Empfehlung zum Kauf oder Verkauf von Finanzinstrumenten oder zum Tätigen irgendwelcher Anlagegeschäfte oder sonstiger Transaktionen dar. Diese Studie (einschließlich der darin enthaltenen Informationen und Meinungen) stellt keine Anlageberatung dar und sollte nicht als solche aufgefasst werden. Potentielle Investoren sind gehalten, spezifische Beratung einzuholen und Anlageentscheide gestützt auf ihre individuellen Anlageziele sowie ihre finanziellen und steuerlichen Gegebenheiten zu treffen.

\section{HAFTUNGSAUSSCHLUSS}

Die Autoren sind darum bemüht, dass diese in dieser Studie enthaltenen Informationen zum Zeitpunkt ihrer Veröffentlichung richtig und vollständig sind und aus zuverlässigen Quellen stammen. Die Autoren lehnen jedoch jegliche Verantwortung für die Genauigkeit, Zuverlässigkeit, Aktualität und Vollständigkeit der hierin wiedergegebenen Informationen und Meinungen ab. Die Autoren lehnen ausdrücklich jegliche Haftung für Verluste oder Schäden ab, die sich aus der Nutzung dieser Studie oder dem Vertrauen in die darin enthaltenen Informationen ergeben könnten, einschließlich Gewinnausfälle oder anderer direkter und indirekter Schäden. 


\section{Mietspiegel aus ökonomischer Sicht - \\ Vorschläge für eine Neuregulierung ${ }^{1}$}

Dr. Oliver Lerbs, Zentrum für Europäische Wirtschaftsforschung (ZEW) und

Prof. Dr. Steffen Sebastian, Universität Regensburg und ZEW

IREBS Beiträge zur Immobilienwirtschaft, Heft 10, 2015

\footnotetext{
${ }^{1}$ Die Autoren danken für zahlreiche Hinweise und Anregungen. Für besonders ausführliche Hinweise danken wir Ulf Börstinghaus (Universität Bielefeld), Walter Krämer (Universität Dortmund), Kristof Dascher, Herbert Grziwotz, Tobias Just, Jürgen Kühling, Walter Oberhofer (alle Universität Regensburg) und Manfred Stelter (ö.b.u.v Sachverständiger, Berlin). Für die inhaltlichen Aussagen sind allein die Autoren verantwortlich.
} 


\section{Inhalt}

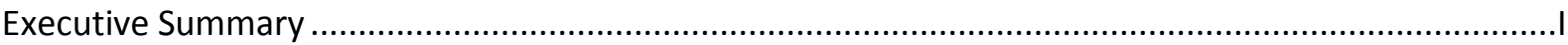

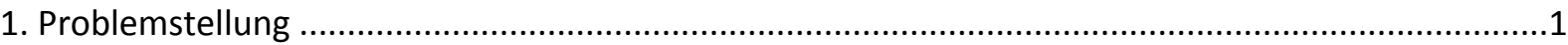

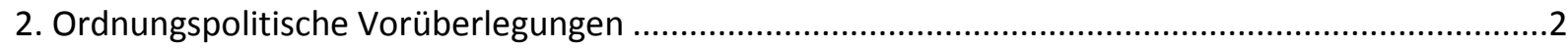

2.1 Grundsätze der Wettbewerbsökonomik .............................................................................. 2

2.2 Gesamtwirtschaftliche Bedeutung funktionsfähiger Mietwohnungsmärkte................................ 4

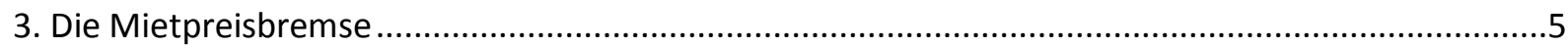

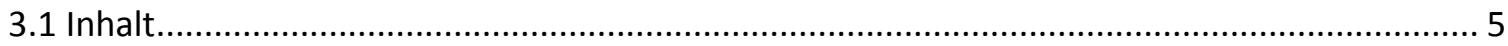

3.2 Zusammenwirken mit dem aktuellen Mietspiegel.............................................................. 5

3.3 Auswirkungen bei mangelnder Mietspiegelreform ……................................................ 7

4. Vorschläge für eine Neuregulierung des (qualifizierten) Mietspiegels ...............................................7

4.1 Berücksichtigung von Neuvermietungen, Mieterhöhungen und Staffelmieten ........................ 8

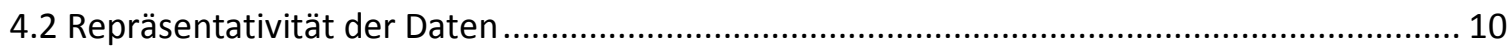

4.3 Berücksichtigung besonderer Anbietergruppen sowie Mieter-Vermieter-Verhältnis............ 13

4.4 Verbesserung des Umgangs mit Unterschieden im Bezugszeitraum der Daten...................... 14

4.5 Abbildung der Lage durch räumliches Regressionsmodell..................................................... 15

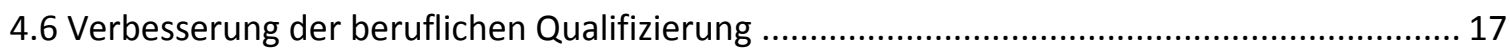

4.7 Stärkung der Dokumentationspflichten ..................................................................... 17

4.8 Unabhängigkeit von politischer Einflussnahme …............................................................... 18

4.9 Keine Detailvorgaben zu Datenaufbereitung und Modellspezifikation .................................. 19

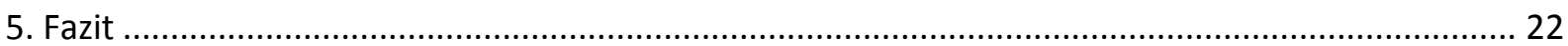

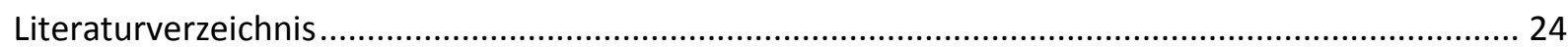




\section{Executive Summary}

Durch die Mietpreisbremse wird die ortsübliche Vergleichsmiete zum zentralen Steuerungselement des Wohnungsmarktes. Konsequenterweise beabsichtigt die Bundesregierung eine Novellierung der gesetzlichen Regelungen zum qualifizierten Mietspiegel.

In dieser gemeinsamen Studie von ZEW und IREBS werden die Anforderungen an korrekte Mietspiegel aus wissenschaftlicher Sicht dargelegt und Vorschläge für eine verbesserte Regulierung entwickelt.

\section{1) Trennung von Statistik und Politik}

In einem Rechtsstaat werden amtliche Statistiken grundsätzlich von einer neutralen Stelle errechnet. Im Gegensatz dazu unterliegt der Mietspiegel trotz seiner hohen Bedeutung zu einem großen Teil dem Gestaltungswillen lokaler Politiker und Interessensvertreter, dessen Umsetzung zudem weitgehend intransparent ist. Dies widerspricht grundlegenden Prinzipien für amtliche Statistiken eines Rechtsstaates. Im Ergebnis sind die durch Mietspiegel errechneten ortsüblichen Vergleichsmieten deutlich niedriger, als sie nach aktuellen Marktbedingungen sein müssten.

Um die Funktionsfähigkeit des Wohnungsmarktes aufrecht zu erhalten, muss der Mietspiegel wieder zu einem marktnahen Abbild des Wohnungsmarktes werden. Eine politische Einflussnahme auf die Berechnung von Mietspiegeln sollte nicht mehr möglich sein.

Sofern eine Begrenzung der Miethöhe politisch erwünscht ist, muss dies getrennt von der Berechnung des Mietspiegels umgesetzt werden. Die Mietpreisbremse kann hier als Vorbild dienen: bei Wiedervermietungen nicht mehr als zehn Prozent über der ortsüblichen Vergleichsmiete. Eine entsprechende Regelung für Mieterhöhungen würde dann noch fehlen. Ob hier ein Abschlag von null, zehn oder dreißig Prozent zur ortsüblichen Vergleichsmiete umgesetzt wird, bleibt allein der Politik vorbehalten.

\section{2) Vollerhebung statt Befragung}

Der Datenumfang in vielen als qualifiziert ausgewiesenen Mietspiegeln ist für eine repräsentative Stichprobe zu gering. Zudem bestehen vielfach Bedenken bzgl. systematischer Verzerrungen bei der Erhebung, etwa aufgrund selektiven 
Antwortverhaltens bei Mieter- und Vermieterbefragungen. Aus methodischen Gründen, aber auch aufgrund der deutlich geringeren Kosten erscheint eine internetbasierte Vollerhebung aller Vermietungsdaten geboten. Auf eine Erfassung von Mieterhöhungen und Staffelmieten sollte hingegen zukünftig verzichtet werden, da diese keinen Bezug zum aktuellen Marktgeschehen aufweisen.

3) Verbreiterung des Datenbasis

Die aktuell praktizierte einfache Durchschnittsbildung ohne Berücksichtigung des unterschiedlichen Alters der Daten ist ein eindeutiger Verstoß gegen grundlegende statistische und wissenschaftliche Prinzipien und in dieser Form bei einer Ausweitung auf zehn Jahre nicht tragbar. Das unterschiedliche Alter der Daten muss vielmehr methodisch explizit berücksichtigt werden. Nur unter diesen Voraussetzungen ist eine Ausweitung des Erhebungszeitraums auf zehn Jahre oder mehr nicht zu beanstanden.

\section{4) Berücksichtigung der Lage}

Die Lage einer Wohnung hat wesentlichen Einfluss auf den Mietpreis und ist damit eines der wichtigsten Wohnungsmerkmale. Die bislang praktizierte Berücksichtigung der Lage ist nur bedingt objektiv und entspricht nicht dem Stand der Forschung. Der Einfluss der Lage sollte durch moderne Methoden der räumlichen Statistik unter Verwendung von Geokodierungen erfasst werden.

\section{5) Transparenz}

Eine Vielzahl der als qualifiziert ausgewiesenen Mietspiegel erfüllt nicht übliche wissenschaftliche Anforderungen an Transparenz in Datenerhebung, Datenaufarbeitung und Datenverarbeitung sowie Offenlegung der Berechnungsergebnisse. Alle Bearbeitungsschritte und Ergebnisse sollten so detailliert dokumentiert sein, dass eine Neuberechnung zur Kontrolle möglich ist.

6) Unabhängige und qualifizierte Berechnung

Mietspiegel sollten nur durch wissenschaftlich ausgebildete und geprüfte Mietspiegel-Statistiker/-innen erstellt werden. Zusätzlich sollte die Zuständigkeit für Ausschreibung und Vergabe der Berechnung von Mietspiegeln bei den Oberen Gutachterausschüssen liegen, um Unabhängigkeit von politischer Einflussnahme zu gewährleisten. 


\section{Problemstellung}

Die Mietpreisbremse ist kontrovers diskutiert worden und hat insbesondere von Seiten der Wissenschaft deutliche Kritik erfahren. ${ }^{2}$ Diese Kritik ging jedoch davon aus, dass sich die Mietpreisbremse an der ortsüblichen Vergleichsmiete orientiert, wie sie durch die bisherigen Mietspiegel berechnet wird. ${ }^{3}$ Es war aber immer die erklärte Absicht des Gesetzgebers, auch die gesetzliche Grundlage für die Berechnung von Mietspiegeln zu überarbeiten. ${ }^{4}$ Konkret heißt es hierzu im Koalitionsvertrag: „Wir sorgen dafür, dass im Mietspiegel die ortsübliche Vergleichsmiete auf eine breitere Basis gestellt und realitätsnäher dargestellt wird. ${ }^{\text {"5 }}$

Die rechtlichen Regelungen zur Berechnung von Mietspiegeln ermöglichen aktuell einen intransparenten Eingriff in den Wohnungsmarkt. Hingegen ist die Mietpreisbremse - bei aller gerechtfertigten Kritik aus ökonomischer Sicht - eine transparente Regulierung. Sofern es gelingt, nunmehr auch die Erstellung von Mietspiegeln adäquat zu regulieren, könnte im Ergebnis eine deutliche Verbesserung der Regulierungspraxis erreicht werden. Durch marktgerechte, wissenschaftlich fundierte Mietspiegel würden zudem mögliche negative Effekte der Mietpreisbremse auf den Wohnungsmarkt minimiert. Ein wesentlicher Teil der Kritik an der Mietpreisbremse würde damit gegenstandslos werden.

Wesentliches Ergebnis der Mietpreisbremse ist, dass nunmehr bei Wiedervermietungen nur noch eine Miete vereinbart werden darf, die maximal zehn Prozent über der sogenannten ortsüblichen Vergleichsmiete gemäß $\S 558$ Abs. 2 BGB liegt ( $\S 556 d$ Abs. 1 BGB). Voraussetzung ist, dass die Wohnung in einem Gebiet mit angespanntem Wohnungsmarkt liegt ( $\$ 556 \mathrm{~d}$ Abs. 2 BGB). ${ }^{6}$ Die ortsübliche Vergleichsmiete wird in der Regel anhand eines Mietspiegels, insbesondere anhand eines qualifizierten Mietspiegels, konkretisiert. Ein qualifizierter Mietspiegel ist gemäß § 558d Abs. 1 BGB nach wissenschaftlichen Grundsätzen zu erstellen.

Der Mietspiegel bestimmt nunmehr nahezu allein den Preis auf dem Mietwohnungsmarkt und wird damit auch zum zentralen Element für die Bildung von Angebot und Nachfrage. Die aktuelle gesetzliche Grundlage zur Erstellung von Mietspiegeln, insbesondere auch von qualifizierten Mietspiegeln nach $\S 558 \mathrm{~d}$ BGB, ist wenig präzise und wird der nochmals gesteigerten Bedeutung des Mietspiegels nicht gerecht. In diesem Aufsatz werden Vorschläge für eine verbesserte Regulierung des qualifizierten Mietspiegels erarbeitet.

\footnotetext{
${ }^{2}$ Vgl. Sachverständigenrat zur Begutachtung der gesamtwirtschaftlichen Entwicklung (2013), S. 463-475.

${ }^{3}$ Eine exakt bestimmbare ortsübliche Vergleichsmiete existiert grundsätzlich nicht. In Mietspiegeln werden daher auch stets Mietpreisspannen angegeben, um zu berücksichtigen, dass selbst weitgehend identische Wohnungen zu unterschiedlichen Mietpreisen vermietet sein können. Aus Vereinfachungsgründen wird im Folgenden dennoch die Bezeichnung ortsübliche Vergleichsmiete verwendet.

${ }^{4}$ Vgl. Bundesregierung (2013), S. 115.

${ }^{5}$ Vgl. Bundesregierung (2013), S. 81.

${ }^{6}$ Nach dem 1.10.2014 fertiggestellte Neubauwohnungen sind ebenso wie die Erstvermietung bei umfassenden Modernisierungen von dieser Regelung ausgenommen (556 f BGB).
} 


\section{Ordnungspolitische Vorüberlegungen}

\subsection{Grundsätze der Wettbewerbsökonomik}

Ein funktionsfähiger Preismechanismus und Vertragsfreiheit gehören in der Sozialen Marktwirtschaft zu den konstituierenden Prinzipien der Wettbewerbsordnung. ${ }^{7}$ Auf dem Mietwohnungsmarkt hat der Mietpreis - sofern er die lokalen Marktverhältnisse korrekt widerspiegelt und nicht künstlich verzerrt ist - eine zentrale Signal- und Lenkungsfunktion. Dies gilt sowohl bei Erstvermietungen von Neubauwohnungen als auch bei Wiedervermietungen von Bestandswohnungen: Um volkswirtschaftlich sinnvolle Entscheidungen zu gewährleisten, muss der Staat in beiden Fällen garantieren, dass bei Vermietungen marktgerechte Mieten verlangt werden können. ${ }^{8}$ Den Haushalten liefert ein marktgerechter Mietpreis Informationen über die Kosten einer Wohnung an einem bestimmten Standort relativ zu möglichen Alternativen. Investoren zeigt er an, welche Arten von Wohnungen in welchen Wohnlagen knapp sind. Beides zusammen gewährleistet, dass

1) Haushalte die bestmögliche Wohnung anmieten, die sie sich ökonomisch leisten können ${ }^{9}$ und

2) Wohnungen in den Lagen und mit den Eigenschaften gebaut werden, die Verbraucher am meisten wertschätzen. ${ }^{10}$

Bislang konnte sich bei Wiedervermietungen die Höhe der vereinbarten Miete an den tatsächlichen Marktverhältnissen orientieren. Durch die Mietpreisbremse darf die zulässige Miete nunmehr maximal zehn Prozent über der sogenannten „ortsüblichen Vergleichsmiete“ liegen. Als ortsüblich gelten dabei gemäß $\S 558$ Abs. 2 BGB diejenigen Entgelte, die in der Gemeinde oder einer vergleichbaren Gemeinde für Wohnraum vergleichbarer Art, Größe, Ausstattung, Beschaffenheit und Lage einschließlich der energetischen Ausstattung und Beschaffenheit in den letzten vier Jahren vereinbart oder geändert worden sind.

Ob und inwieweit die Mietpreisbremse langfristig negative volkswirtschaftliche Effekte entfalten wird, wird entscheidend davon abhängen, ob und inwieweit die zur Bestimmung des ortsüblichen Vergleichsmietniveaus maßgeblichen Mietspiegel das lokal gegebene Marktmietniveau widerspiegeln. Bislang ist die ortsübliche Vergleichsmiete ein Instrument, das in bestehenden Mietverträgen eine Orientierung für die Angemessenheit von Mieterhöhungen geben, damit soziale Härten verhindern ${ }^{11}$ und Rechtssicherheit schaffen soll. ${ }^{12}$ Die ortsübliche Vergleichsmiete ist aber

\footnotetext{
${ }^{7}$ Vgl. Eucken (1952), S. 254-291.

${ }^{8}$ Vgl. Eekhoff (2002), S. 38-39; Sachverständigenrat zur Begutachtung der gesamtwirtschaftlichen Entwicklung (2013), S. 463.

${ }^{9}$ Eine Soziale Marktwirtschaft ergänzt und stärkt dieses Grundprinzip durch eine soziale Absicherung des Wohnens. Haushalten, die über ein geringes Einkommen verfügen oder sich aus anderen Gründen nicht am Markt adäquat mit Wohnraum versorgen können, ist anhand geeigneter Maßnahmen öffentlich finanzierte und koordinierte Unterstützung zu leisten.

${ }^{10}$ Vgl. Eekhoff (2002), S. 40.

${ }^{11}$ Die Regulierung von Mieterhöhungen in laufenden Mietverträgen ist sowohl aus juristischer als auch aus institutionenökonomischer Sicht äußerst sinnvoll, da sie eine sonst mögliche Umgehung des gesetzlichen Kündigungsschutzes durch die Forderung des Vermieters, das Mietverhältnis unter überzogener Mieterhöhung fortzusetzen, verhindert. Für eine institutionenökonomische Diskussion dieses sog. „Hold-up“ vgl. Richter und Furubotn (2003), S. 370.
} 
gerade nicht ein Spiegel des aktuellen Marktgeschehens bei Wiedervermietungen. Durch die Mietpreisbremse erhält die ortsübliche Vergleichsmiete plötzlich eine ökonomisch wichtige Allokationsfunktion. Diese wird sie nur dann erfüllen können, wenn mit dem Mietspiegel das entscheidende Instrument ihrer Ermittlung neu und „realitätsnäher" - im Sinne von marktnäher reguliert wird.

Eine staatliche Preisregulierung bei Wiedervermietungen, die nicht auf eine adäquat ermittelte, marktorientierte Vergleichsmiete Bezug nimmt, wäre ordnungspolitisch lediglich dann gerechtfertigt, wenn dieser Markt systematisch durch Monopolisierungstendenzen oder Marktversagen infolge externer Effekte, Informations- oder schwerwiegender Anpassungsmängel geprägt wäre. ${ }^{13}$ Dies ist jedoch nicht der Fall: Gerade in Deutschland gewährleisten unterschiedliche Eigentümergruppen und zahlreiche Einzeleigentümer ein differenziertes Angebot. Markteintritt und -austritt sind möglich, ohne dass die dazu aufzuwendenden Investitionen irreversibel sind. Konkurrenz ist also für alle Anbieter unmittelbare Realität. Externe Effekte sind unter Einhaltung von Bauordnungen und Flächennutzungsplänen nicht zu erwarten. ${ }^{14}$ Mieter und Vermieter können sich vor Vertragsabschluss über die tatsächliche Qualität einer Wohnung und die Bonität des Vertragspartners informieren. Auch schwerwiegende Anpassungsmängel liegen bisher nicht vor: Zwar erfordert eine spürbare Angebotsausweitung aufgrund technischer Gegebenheiten eine gewisse Zeit, jedoch zeigt der starke Anstieg der Baufertigstellungen gerade im Geschosswohnungsbau der letzten Jahre, dass der Wohnungsneubau bisher angemessen auf Nachfragesteigerungen reagieren konnte.

Die positiven Auswirkungen einer marktgerechten Preisbildung auf das Angebot lassen sich sehr treffend am Beispiel des Büroimmobilienmarktes verdeutlichen. Wie der Wohnungsmarkt auch, muss der Büroimmobilienmarkt auf das naturgemäß begrenzte Angebot an Bauland zurückgreifen. Zudem wird der Wohnungsbau, bspw. bei der Ausweisung von innerstädtischem Bauland, regelmäßig zulasten des Büroimmobilienmarktes privilegiert. Dennoch hat die Bildung marktgerechter Preise auf dem Büroimmobilienmarkt zu dem gleichen Phänomen geführt, wie es in modernen Volkswirtschaften bei vielen Konsum- und Investitionsgütern zu beobachten ist: Die Anbieter produzieren weitaus mehr, als am Markt nachgefragt wird. Das resultierende Überangebot drückt zugunsten des Nachfragers den Preis. Im Büroimmobilienmarkt zeigt sich dies inzwischen durch erheblichen Leerstand und eine gestiegene Verhandlungsmacht der Mieter bei der Festsetzung des Mietpreises und der Ausstattung. Demgegenüber haben Bemühungen des Gesetzgebers, in den Mietwohnungsmarkt preisregulierend einzugreifen, nicht nur dazu geführt, dass in den vielen Ballungsgebieten ein Nachfrageüberschuss nach Wohnungen besteht, der derzeit nicht befriedigt werden kann. Zudem sind an vielen Standorten inzwischen Wohnflächen teurer geworden als Büroflächen. In der Folge werden aktuell in Ballungsgebieten (u. a. Berlin, Frankfurt, München) unter

\footnotetext{
${ }^{12}$ Anders als der Mietpreis bei Wiedervermietung sind Mieterhöhungen in laufenden Bestandsmietverträgen bereits seit der Einführung der Wohnraumkündigungsschutzgesetze in den 1970er Jahren im deutschen Mietrecht reguliert. Gleichwohl hat der Gesetzgeber erst im Zuge der Mietrechtsreform von 2002 den Begriff der ortsüblichen Vergleichsmiete in das Gesetz aufgenommen.

${ }^{13} \mathrm{Vgl}$. Fritsch et al (2006), S. 80-99, 159-204, 245-289, 289-315.

${ }^{14}$ Eine Ausnahme bietet struktureller Wohnungsleerstand, der mit negativen externen Effekten auf umliegende Immobilien verbunden sein kann, vgl. Eekhoff (2002), S. 32-35 sowie S. 211-212. Das Problem dauerhafter Wohnungsleerstände aufgrund strukturellen Nachfragemangels tritt allerdings in lokalen Wohnungsmärkten auf, für die die Mietpreisbremse keine Relevanz hat.
} 
erheblichem finanziellen Aufwand Büro- und andere Nichtwohnimmobilien in Wohnungen umgewandelt, da sich hier mittlerweile höhere Renditen erzielen lassen. ${ }^{15}$ Zumindest in bestimmten Ballungszentren zeigt sich somit empirische Evidenz, dass eine marktgerechte Preisbildung für die Mieter das bessere Angebot - in Form von niedrigeren Mieten - schafft als die regulierenden Eingriffe in die Preisbildung am Wohnungsmarkt.

\subsection{Gesamtwirtschaftliche Bedeutung funktionsfähiger Mietwohnungsmärkte}

Der Existenz eines funktionsfähigen Mietwohnungsmarktes mit marktgerechter Preisbildung kommt eine wichtige gesamtgesellschaftliche Stabilitätsfunktion zu, wie nicht zuletzt durch die SubprimeKrise in den USA deutlich geworden ist. Die Wohneigentumsquote in Deutschland ist eine der niedrigsten in Europa. Aus ökonomischer Sicht ist dies durchaus positiv zu bewerten; sowohl für Mieter als auch für die Volkswirtschaft als Ganzes. Die niedrige Wohneigentumsquote ist vor allem dadurch begründet, dass in Deutschland das Wohnen zur Miete gerade aufgrund des differenzierten Marktangebots ein sehr wettbewerbsfähiges Substitut zum Eigentum ist. ${ }^{16}$ Insbesondere finanziell langfristig schwächer gestellte Haushalte dürfen durch Überregulierung des Mietwohnungssektors und Subventionierung des Eigentums nicht künstlich in den Wohneigentumssektor gedrängt werden, wie es in einer Vielzahl anderer Länder der Fall ist. Dies würde zu einer für diesen Bevölkerungskreis übermäßig riskanten Allokation von Krediten führen und wäre auch hinsichtlich der Finanzmarktstabilität bedenklich.

Zur Sicherstellung einer angemessenen und preisgünstigen Versorgung der Bevölkerung mit Wohnraum darf die Preisbildung auf dem Mietwohnungsmarkt nicht übermäßig eingeschränkt werden. ${ }^{17}$ Eine künstlich überhöhte Wohneigentumsquote ist oft das negative Ergebnis eines nicht funktionierenden Mietwohnungsmarktes. Wenn Vermietung sich nicht mehr lohnt, geht das Angebot an privat finanzierten Mietwohnungen zurück und Wohnungssuchende können nur noch auf dem Wege des schuldenfinanzierten Eigentumserwerbs oder durch öffentlich finanzierten Wohnungsbau zu Wohnraum kommen. So ist es in vielen Städten in Skandinavien, Großbritannien und den USA aufgrund der Überregulierung der Märkte zu erheblichen Engpässen auf den privaten Mietwohnungsmärkten gekommen. In einer Vielzahl von Ländern, insbesondere in Schweden, Spanien und den Niederlanden, werden daher aktuell Deregulierungen insbesondere des öffentlich geförderten Mietwohnungsmarktes vorgenommen und zugleich Subventionierungen des Wohneigentums eingeschränkt, um die Funktionsfähigkeit der Wohnungsmärkte wieder herzustellen. ${ }^{18}$

Es ist daher insbesondere im Zusammenhang mit der Mietpreisbremse erforderlich, dass die zur Festlegung der Miethöhe relevante ortsübliche Vergleichsmiete an den tatsächlichen lokalen Marktverhältnissen verankert wird und nicht wie bislang systematisch nach unten verzerrt ist. Über Mieten bei Wiedervermietungen hinaus sollten sich auch Mieterhöhungen grundsätzlich an der aktuellen Marktmiete orientieren, damit der Mietpreis auch für die bereits vermieteten Wohnungen - und damit für den überwiegenden Anteil des Wohnungsbestandes - seine Funktion der Steuerung von Standortentscheidungen und Neuinvestitionen beibehält. Anderenfalls ist $u$. a. damit zu rechnen,

\footnotetext{
${ }^{15} \mathrm{Vgl}$. Bundesministerium für Verkehr, Bau- und Wohnungswesen (2013).

${ }^{16}$ Vgl. Lerbs (2014), S. 38.

${ }^{17}$ Vgl. Sachverständigenrat zur Begutachtung der gesamtwirtschaftlichen Entwicklung, Hrsg. (2013), S. 465-66.

${ }^{18}$ Andrews et al. (2011), S. 10 sowie S. 12-14; Patrizia Immobilien AG (2014), S. 20-27.
} 
dass mit dem knappen Gut Wohnraum ineffizient umgegangen wird, beispielsweise wenn Einzelpersonenhaushalte entgegen ihren Bedarfswünschen in großen Wohnungen verbleiben, weil die Neuanmietung einer kleineren Wohnung deutlich teurer wäre als die aktuell gezahlte, preisregulierte Miete. Der Bedarf anderer Marktteilnehmer nach großen Wohnungen, bspw. von Familien, kann dann aufgrund der regulierungsbedingten Marktverzerrung deutlich schlechter berücksichtigt werden.

\section{Die Mietpreisbremse}

\subsection{Inhalt}

Infolge des Mietrechtsnovellierungsgesetzes (MietNovG) ist vorgeschrieben, dass in vorab durch die Landesregierungen per Rechtsverordnung auszuweisenden Gebieten („,angespannte Wohnungsmärkte“) im Fall der Wiedervermietung von Bestandswohnungen die zulässige Miete maximal zehn Prozent über der ortsüblichen Vergleichsmiete liegen darf. Damit hat der Gesetzgeber die Regulierung von Mieterhöhungen ( $\$ 558$ Abs. 1 BGB) auf die Wiedervermietung von Bestandswohnungen erweitert. Qualifizierend gilt zu beachten, dass

- Wohnungen, die nach dem 1. Oktober 2014 erstmalig genutzt und vermietet werden, von der Regelung ausgenommen sind ( $\$ 556 f$ Satz 1 BGB),

- ebenso die erste Vermietung nach umfassender Modernisierung, nicht aber alle folgenden Vermietungen (556f Satz 2 BGB) und

- in Fällen, bei denen die bisher vereinbarte Miete über der ortsüblichen Vergleichsmiete zuzüglich zehn Prozent liegt, der Vermieter diese Miete auch weiterhin verlangen darf, die Miete also nicht senken muss (§556e BGB).

Analog zur Regulierung der Miete in laufenden Mietverträgen kommt der ortsüblichen Vergleichsmiete bei der Mietpreisbremse eine entscheidende Bedeutung zu. Ihre Höhe und die Glaubwürdigkeit ihrer Bestimmung werden die ökonomische „Bremswirkung“ und damit die Kosten der Regulierung maßgeblich beeinflussen.

\subsection{Zusammenwirken mit dem aktuellen Mietspiegel}

Nicht gesetzlich vorgeschrieben ist, wie genau die ortsübliche Vergleichsmiete zu bilden ist. Es handelt sich somit um einen unbestimmten Rechtsbegriff, der näherer inhaltlicher Bestimmung bedarf. In der Praxis hat sich infolgedessen eine Vielfalt von Vorgehensweisen entwickelt. ${ }^{19}$ Dem seit dem Jahr 2002 in $\S 558$ d BGB näher definierten qualifizierten Mietspiegel kommt dabei eine besondere Bedeutung $\mathrm{zu}$, denn dieser gilt de lege lata als „nach anerkannten wissenschaftlichen Grundsätzen erstellt" ( $§ 558 \mathrm{~d}$ Abs. 1 BGB) und erhält die gesetzliche Vermutung, die ortsübliche Vergleichsmiete bei regelmäßiger Anpassung gemäß §558d Abs. 2 BGB wiederzugeben.

Auf der Grundlage der aktuellen Berechnungspraktiken ist davon auszugehen, dass die ortsüblichen Vergleichsmieten die tatsächlichen Knappheitsverhältnisse an städtischen Wohnungsmärkten völlig unzureichend widerspiegeln. Nach Untersuchungen des Bundesinstituts für Bau-, Stadt- und

\footnotetext{
${ }^{19}$ Diese reichen vom einfachen oder qualifizierten Mietspiegel bis hin zu begründeten Sachverständigengutachten oder Entgelten für drei einzelne, als vergleichbar benannte Wohnungen.
} 
Raumforschung (BBSR) liegt die ortsübliche Vergleichsmiete in der Mehrheit der Fälle deutlich (bis zu 42 Prozent) unter der Angebotsmiete, die aus Zeitungs- und Internetannoncen zu ermitteln ist und als geeigneter Indikator für tatsächlich vertraglich vereinbarte Neu- und Wiedervermietungsmieten gelten kann. Abweichungen nach oben kommen hingegen nicht vor. ${ }^{20}$

Sofern das bisherige Erstellungskonzept der Mietspiegel beibehalten wird, handelt es sich beim verabschiedeten Konzept der Mietpreisbremse nicht lediglich um die gewünschte Begrenzung der dynamischen Mietentwicklung, also eine Mietpreiswachstumsbremse, ${ }^{21}$ sondern faktisch um eine marktwirksame, d. h. ökonomisch bindende Preisabsenkung.

Die fehlende Überdeckung von Marktmiete und ortsüblicher Vergleichsmiete widerspricht der ursprünglichen Intention des Gesetzgebers, der das Konzept der ortsüblichen Vergleichsmiete bei dessen Einführung als weitgehend marktorientierte Miete interpretiert hat. ${ }^{22}$ Diesem Verständnis liegt die ökonomisch zutreffende Einsicht zugrunde, dass Mietpreise ihre Signal- und Lenkungsfunktion grundsätzlich nur dann erfüllen können, wenn sie marktgerecht sind. Eine Miete kann als marktgerecht verstanden werden, wenn sich Mieter und Vermieter im gewöhnlichen Geschäftsverkehr, d. h. unter offenem Markt- und Informationszugang, persönlicher Freiheit und zeitaktuell auf diese Miete geeinigt haben. ${ }^{23}$ Dieser Definition entgegenstehend hat die ortsübliche Vergleichsmiete heute neben einer empirischen eine stark politisch motivierte, normative Komponente. So wird sie üblicherweise als "modifizierte marktorientierte Durchschnittsmiete“ interpretiert. ${ }^{24}$ Dieses Verständnis wird den neuen Anforderungen, die im Zuge der Mietpreisbremse an die ortsübliche Vergleichsmiete erwachsen, nicht gerecht.

(Nur) in Kombination mit den aktuell nicht marktgerechten ortsüblichen Vergleichsmieten wird die Mietpreisbremse bewirken, dass Vermieter bei Wiedervermietungen auf Dauer keine Marktmieten mehr verlangen können. In einer wachsenden Volkswirtschaft mit positiver Preissteigerungsrate ist dies aus ökonomischer Sicht mit einer Zusatzbesteuerung gleichzusetzen, d. h. die Mietpreisbremse ist eine Sondersteuer auf Mieteinnahmen. Allerdings kommt diese Form der Besteuerung nicht der Allgemeinheit zugute. Letztlich profitiert hiervon nur der Mieter der jeweiligen Wohnung unabhängig von dessen Bedürftigkeit und auch, wenn er finanziell besser gestellt ist als der Vermieter. Zudem wird diese Steuer unabhängig von der wirtschaftlichen Leistungsfähigkeit des Vermieters erhoben, trifft also "arme" und "reiche" Vermieter in gleicher Weise. Es ist sehr fragwürdig, ob diese Form der Umverteilung effizient und gerecht ist. ${ }^{25}$ Eine Abbildung der

\footnotetext{
${ }^{20} \mathrm{Vgl}$. Held et. al (o. J.), S. 6, $26 \mathrm{f}$.

${ }^{21}$ Vgl. Turner und Malpezzi (2011), S. 82.

${ }^{22}$ Das Konzept der ortsüblichen Vergleichsmiete hat mehrere gesetzliche Vorläufer. Rechtshistorisch betrachtet ist die Auffassung begründbar, dass das Konzept der ortsüblichen Vergleichsmiete auf die Marktmiete im ökonomischen Sinn Bezug nimmt, vgl. Niederberger (1980), S. 5-15. Zur historischen Entwicklung vgl. Börstinghaus und Clar (2013), S. 1-8; (2014), S. 1-5.

${ }^{23}$ Dieses ökonomisch plausible Verständnis der Marktmiete ist analog zur Legaldefinition des Verkehrswertes nach § 194 Baugesetzbuch. Ebenso schließt dies „Mietwucher“ gemäß § 5 WiStG explizit aus.

${ }^{24}$ Vgl. hierzu Börstinghaus und Clar (2014), S. 5-6.

${ }^{25} \mathrm{Vgl}$. Olsen (1991), S. 935-938. Sofern eine Belastung des Vermieters zugunsten einer Entlastung des Mieters gewünscht ist, wäre eine deutlich gerechtere Ausgestaltung ein Sonderzuschlag bei der Einkommensteuer auf Mieteinnahmen. Dieser könnte dann zur Finanzierung von Sonderzahlungen in Form von Wohngeld an besonders bedürftige Mieter gezahlt werden. Die negativen Anreizeffekte auf den Wohnungsmarkt wären allerdings die gleichen.
} 
tatsächlichen Marktmiete durch die (qualifizierten) Mietspiegel ist somit auch aus Gründen der Verteilungsgerechtigkeit geboten.

\subsection{Auswirkungen bei mangelnder Mietspiegelreform}

Die negativen volkswirtschaftlichen Auswirkungen der Begrenzung des Mietwachstums in bestimmten Segmenten des Wohnungsmarkts - etwa bei Wiedervermietungen, nicht aber beim Neubau - sind zwar nicht so gravierend wie ein Einfrieren sämtlicher Mieten auf Bestandsniveau. Jedoch zeigen empirische Untersuchungen für verschiedene internationale Fallbeispiele deutlich, dass auch die Einführung von dynamischen Mietobergrenzen in einzelnen Marktsegmenten zu Angebotsverlusten und Fehlallokationen von Wohnungen führen kann. Ökonomisch kommt es auf die Höhe des Bezugspunkts an: Führt die Oberbegrenzung aufgrund nicht marktgerechter Vergleichsmieten zu einer bindenden Beschränkung, kommt es sehr wahrscheinlich zu negativen Folgewirkungen. So wurde beispielsweise in Dänemark infolge der dort eingeführten Mietpreisobergrenzen die Wohnflächennachfrage im regulierten Bereich künstlich überhöht und im nicht regulierten Bereich dagegen zu niedrig gehalten, sodass private Investoren aus dem Markt ausschieden. ${ }^{26}$ In den USA war die Einführung von Mietenregulierungen zwar nur mit vergleichsweise geringen Effekten auf den Neubau verbunden, ließ jedoch ebenfalls Eigentümer in großem Ausmaß Wohnungen aus dem Mietmarkt abziehen und bspw. in Eigentumswohnungen umwandeln. Zugleich kam es zu negativen Auswirkungen auf die Qualität im Wohnungsbestand. ${ }^{27}$ In einer weiteren Studie für die USA wird geschätzt, dass infolge der Mietpreisregulierung in New York City etwa ein Fünftel aller Mietwohnungen in dem Sinne fehlbelegt ist, dass kleinere und einkommensstärkere Haushalte die untersuchten Wohnungen bewohnen, als sie es in einem regulierungsfreien Markt täten. ${ }^{28}$

Ähnliche negative Auswirkungen der Mietpreisbremse in Deutschland sind zu erwarten, wenn die durch Mietspiegel ausgewiesenen ortsüblichen Vergleichsmieten nicht die Marktmiete abbilden. Sofern Mietspiegel nach wissenschaftlichen Grundsätzen erstellt werden, sind diese aber durchaus in der Lage, die Marktverhältnisse wiederzugeben und auch dynamische Veränderungen zeitnah abzubilden. Unter diesen Voraussetzungen sind keine schwerwiegenden Verzerrungen des Wohnungsmarktes zu erwarten.

\section{Vorschläge für eine Neuregulierung des (qualifizierten) Mietspiegels}

Bei der Ermittlung der ortsüblichen Vergleichsmiete mittels qualifizierter Mietspiegel fordert der Gesetzgeber gemäß $\S 558 \mathrm{~d}$ BGB ausdrücklich, wissenschaftliche Anforderungen zu beachten, konkretisiert diese aber nicht näher. Werden als Maßstab der Wissenschaftlichkeit etwa die Empfehlungen zur Sicherung guter wissenschaftlicher Praxis der Deutschen Forschungsgemeinschaft (DFG) angelegt, so sind dies u. a. folgende Grundsätze: ${ }^{29}$

- „de lege artis“ zu arbeiten, d.h. insbesondere, alle aktuell bestehenden brauchbaren Erkenntnisse und technischen Möglichkeiten zu berücksichtigen und

- alle Resultate zu dokumentieren und konsequent selbst anzuzweifeln.

\footnotetext{
${ }^{26}$ Vgl. Skak und Bloze (2013), S. 14-16.

${ }^{27} \mathrm{Vgl}$. Sims (2007), S. 140-146.

${ }^{28} \mathrm{Vgl}$. Glaeser und Luttmer (2003), S. 1041-1043.

${ }^{29} \mathrm{Vgl}$. Deutsche Forschungsgemeinschaft (2013), S. 15.
} 
Bereits diese grundlegenden Anforderungen können in der bisherigen Mietspiegelpraxis als kaum erfüllt gelten. ${ }^{30}$ Die Prozesse der Datenerhebung und -auswertung im Rahmen qualifizierter Mietspiegel folgen häufig nicht modernen statistischen Standards und werden zudem nur bedingt ausreichend kommentiert und dokumentiert, was die Nachvollziehbarkeit der Ergebnisse erheblich einschränkt. ${ }^{31}$

Bei einer Beurteilung, ob und inwieweit sich empirisch ermittelte Vergleichswerte für eine gute Schätzung der marktgerechten Miete einer Wohnung eignen, sind aus wissenschaftlicher Perspektive insbesondere folgende Punkte von Bedeutung: ${ }^{32}$

\section{1) Konsistenz der Datenbasis}

- Berücksichtigung von Neuvermietungen, Mieterhöhungen und Staffelmieten

- Repräsentativität der Daten (Vollerhebung statt Stichproben)

- Berücksichtigung spezieller Anbietergruppen

2) Eignung der Berechnungsmethodik

- Umgang mit zeitlichem Unterschied der Daten

- Operationalisierung der wohnwertbestimmenden Merkmale, vor allem der Lage

- Datenaufbereitung und Modellspezifikation

\section{3) Unabhängigkeit und Transparenz}

- Verbesserung der beruflichen Qualifizierung

- Stärkung der Dokumentationspflichten

- Unabhängigkeit von politischer Einflussnahme

Diese Anforderungen aus wissenschaftlicher Sicht zu diesen Punkten werden wir im Folgenden genauer ausführen. Hierbei werden wir auch konkrete Vorschläge für deren operationale Umsetzung erarbeiten.

\subsection{Berücksichtigung von Neuvermietungen, Mieterhöhungen und Staffelmieten}

Von dem Konzept der Marktmiete hat sich die ortsübliche Vergleichsmiete mittlerweile weitgehend entfernt. Es handelt sich hierbei um eine mehr oder minder politisch definierte Größe, die keinen ökonomischen Bezug aufweist und sich somit auch einer ökonomischen Interpretation entzieht. Nach der Legaldefinition des $\S 558$ Abs. 2 BGB ist die ortsübliche Vergleichsmiete ein nicht näher definierter Durchschnitt aus den im Rahmen von Neuvermietungen, Mieterhöhungen oder Staffelmieten vereinbarten Mieten der letzten vier Jahre. ${ }^{33}$ Die derzeitige Regelung lässt jedoch

\footnotetext{
${ }^{30}$ Vgl. Freund et al. (2013). S. 260.

${ }^{31}$ Vgl. Freund et al. (2013), S. 261-263, Krämer (2014), o. S., Schlittgen und Uhlig (1997), S. 314.

${ }^{32}$ Vgl. u. a. Börstinghaus und Clar (2013), S. 167-169, Freund et al. (2013), S. 259, Schlittgen und Uhlig (1997), S. 314.

${ }^{33}$ Dies ist auch Grundlage bei der Berechnung von Mietspiegeln nach §558c BGB bzw. sogenannten qualifizierten Mietspiegeln nach 558d BGB, wobei bei letzteren noch u. a. die Anforderung hinzukommt, dass diese nach anerkannten wissenschaftlichen Grundsätzen erstellt werden müssen.
} 
insofern eine wesentliche Lücke, als dass nicht geklärt ist, welchen Anteil jeweils Neuvermietungen, Mieterhöhungen oder Staffelmieten bei der Berechnung der ortsüblichen Vergleichsmiete haben sollen. Dies lässt den handelnden Parteien einen sehr weiten Konkretisierungsspielraum. ${ }^{34}$ Eine willkürfreie Berechnung der ortsüblichen Vergleichsmiete ist somit gefährdet, wenn nicht sogar unmöglich.

Nur Neuvermietungen konnten nach bisheriger Rechtslage frei verhandelt werden und nur insoweit enthält die ortsübliche Miete einen Bezug zur Marktmiete.

Mieterhöhungen werden hingegen aus ökonomischer Sicht grundsätzlich nicht frei verhandelt, da weder Mieter noch Vermieter die Möglichkeit haben, mit einem anderen Vertragspartner eine Mieterhöhung über die vertragsgegenständliche Wohnung abzuschließen. Die Verhandlungen finden daher nicht unter Wettbewerbsbedingungen statt. So könnte ein Mieter eine eigentlich nicht rechtswirksame Mieterhöhung dennoch akzeptieren, da er den Vertragsfrieden mit dem Vermieter nicht gefährden möchte. Aus dem gleichen Grund könnte ein Vermieter nur eine Miete unterhalb der ortsüblichen Vergleichsmiete verlangen oder auf eine Mieterhöhung ganz verzichten, um ein einvernehmliches Mietverhältnis nicht zu gefährden.

Im Falle eines einseitigen Mieterhöhungsverlangens des Vermieters darf wiederum die zum Zeitpunkt der Mieterhöhung gültige ortsübliche Vergleichsmiete nicht überschritten werden. Diese ortsübliche Vergleichsmiete enthält jedoch teilweise Mieterhöhungen, welche aus einem zurückliegenden Vier-Jahres-Zeitraum stammen. Wird beispielsweise eine Mieterhöhung auf Basis des Mietspiegels 2015 ausgesprochen, so enthält die darin ausgewiesene ortsübliche Vergleichsmiete anteilig auch Mieterhöhungen aus den Jahren 2014-2011. Mieterhöhungen aus dem Jahr 2011 wurden aber damals auf Basis des Mietspiegels 2011 ausgesprochen, der wiederum anteilig Mieterhöhungen aus dem Jahr 2010-2007 enthält etc. Über diesen Mechanismus werden in einem aktuellen Mietspiegel schlussendlich Daten integriert, die bis zum ersten Mietspiegel zurückreichen, der jemals in dieser Gemeinde erstellt wurde (allerdings mit abnehmender Gewichtung). Darüber hinaus können die durch einseitige Mieterhöhungen verlangten Mietpreise im Wert nur unter, niemals aber über der der ortsüblichen Vergleichsmiete liegen. ${ }^{35}$ Geht man von im Zeitablauf steigenden Mietpreisen aus, so bewirkt die Einberechnung von einseitigen Mieterhöhungen in die ortsübliche Vergleichsmiete also eine systematische Verzerrung der Marktmiete um veraltete und marktferne Daten nach unten. Diese Verzerrung kann nur mit politischem Gestaltungswillen begründet werden, der sich einer wissenschaftlichen Beurteilung entzieht.

Etwas differenzierter sieht es bei der Einberechnung von Staffelmieten nach § 557a BGB aus, d. $\mathrm{h}$. bereits bei Abschluss des Mietvertrags vereinbarten Mieterhöhungen. Zwar handelt es sich auch hier um Veränderungen ohne aktuellen Marktbezug. Da die Höhe der Staffelmieten aber grundsätzlich von der ortsüblichen Vergleichsmiete unabhängig ist, können hier im Ergebnis Mieten vereinbart werden, die grundsätzlich über oder unter der Marktmiete liegen. Eine systematische Verzerrung der ortsüblichen Vergleichsmiete ist durch die Einberechnung von Staffelmieten also prinzipiell nicht

\footnotetext{
${ }^{34}$ Vgl. Börstinghaus und Clar (2013), RN 290-291.

${ }^{35}$ Bspw. aufgrund der Kappungsgrenze nach § 558 Abs. 3 BGB.
} 
gegeben. Dennoch ist die Einbeziehung von durch in der Vergangenheit vereinbarten Staffelmieten aufgrund des fehlenden aktuellen Marktbezuges nicht sinnvoll. ${ }^{36}$

Empfehlung: Mieterhöhungen und Staffelmieten spiegeln nicht die aktuellen Marktverhältnisse wider, da diese nicht in einem freien Verhandlungsprozess zwischen den Parteien entstehen. Damit die ortsübliche Vergleichsmiete den Vermietungsmarkt verzerrungsfrei widerspiegeln kann, sollten Mieterhöhungen und Staffelmieten daher bei der Berechnung eines qualifizierten Mietspiegels nicht berücksichtigt werden.

\subsection{Repräsentativität der Daten}

Eine wesentliche Voraussetzung für die Validität qualifizierter Mietspiegel ist, dass die verwendete Datenbasis für die zuvor richtig abzugrenzende Grundgesamtheit aller relevanten Mietwohnungen repräsentativ ist. ${ }^{37}$ Eine Stichprobe ist repräsentativ, wenn sie die Grundgesamtheit, aus der sie gezogen wird, strukturgetreu widerspiegelt. Strukturtreue ist gemäß Stichprobentheorie insbesondere dann zu erwarten, wenn alle Wohnungen der relevanten Grundgesamtheit dieselbe Chance besitzen, in die Stichprobe zu gelangen. ${ }^{38}$ In der bisherigen Mietspiegelpraxis ist dies kaum der Fall. Es ergeben sich im Gegenteil hinsichtlich der Repräsentativität der Datenstichproben erhebliche Probleme. Ein wesentlicher Grund hierfür ist das selektive Antwortverhalten bei den zur Gewinnung erforderlicher Daten heutzutage üblichen Mieter- und Vermieterbefragungen, durch die mietspiegelrelevante Wohnungen letztlich unterschiedlich hohe Wahrscheinlichkeiten haben, Bestandteil der zur Ermittlung des Mietspiegels maßgeblichen Nettostichprobe zu werden. ${ }^{39}$

Das heute gängige Verfahren befragungsbasierter Stichprobenerhebungen sollte daher durch eine vollständige Erfassung aller Neuvermietungen mit deren wesentlichen Eigenschaften in einem elektronischen Register ersetzt werden (Vollerhebung). Eine derartige Vollerhebung könnte z.B. erreicht werden, indem Vermieter zur Meldung aller mietspiegelrelevanten Informationen verpflichtet werden. ${ }^{40}$ Hierzu müssten Meldemöglichkeiten mittels internetbasierter sowie eventuell auch maschinenlesbarer Formulare zur Verfügung gestellt werden. ${ }^{41}$

\footnotetext{
${ }^{36}$ Hingegen kann die Vereinbarung einer Staffelmiete ein wesentlicher Vertragsbestandteil sein, der bei der Vermietung selbst als wesentliches Merkmal des Mietvertrages Berücksichtigung finden kann.

${ }^{37}$ Vgl. bereits Schlittgen und Uhlig (1997), S. 314.

38 Über die reine Zufallsstichprobe hinaus bestehen mit dem Quoten- und Klumpenstichprobenverfahren weitere Stichprobenverfahren, die für die Ziehung statistisch repräsentativer Stichproben herangezogen werden können.

${ }^{39}$ Vgl. hierzu Krämer (2014), o. S.; Bundesministerium für Verkehr, Bau und Stadtentwicklung (2002), S. 36-37.

${ }^{40} \mathrm{Im}$ Gegensatz zu Mietdatenbanken nach $\S 558$ e BGB, bei denen die Meldung von Daten auf freiwilliger Basis erfolgt. Zu den Möglichkeiten der Datenbeschaffung bei Mietdatenbanken vgl. Stöver (2002), S. 67 f.

${ }^{41}$ Viele der zur Erstellung von qualifizierten Mietspiegeln benötigten Informationen werden von Vermietern heute bereits regelmäßig anlässlich der Vermietung bei einschlägigen Internetplattformen eingespeist. Die Anbieter derartiger Marktplätze würden durch eine Registrierungspflicht der Vermietungsdaten voraussichtlich motiviert werden, diese als integrierten Teil ihrer Dienstleistung anzubieten, wodurch sich der Aufwand für die Vermieter deutlich reduzieren könnte. Bei der Umsetzung sind datenschutzrechtliche Aspekte zu beachten.
} 
Der möglichen Kritik, dass eine solche Vollerhebung ein unangemessener Aufwand wäre, muss zum einen entgegnet werden, dass die Mietpreisbremse einen derart erheblichen Eingriff in die Vertragsfreiheit darstellt, dass sogar deren Vereinbarkeit mit dem Grundgesetz in Frage gestellt wird. ${ }^{42} \mathrm{Im}$ Verhältnis zu diesem wesentlichen Eingriff in die Grundrechte der Eigentümer muss eine Vollerhebung nicht nur als angemessen gelten, sondern ist unseres Erachtens eine notwendige Bedingung für eine adäquate Umsetzung der Mietpreisbremse. In Gebieten, in denen die Mietpreisbremse neu eingeführt wird, sollte daher auch rückwirkend für die letzten zwei Jahre eine Vollerhebung durchgeführt werden, um die erforderliche rechtssichere Basis für die Durchführung der Mietpreisbremse zu schaffen. Damit wäre auch eher zu gewährleisten, dass die Mietpreisbremse grundgesetzkonform ist.

Zum anderen sind wir der Auffassung, dass eine Vollerhebung sowohl organisatorisch als auch finanziell einen deutlich geringeren Aufwand als eine Befragungserhebung beinhaltet. ${ }^{43}$ Es ist zu erwarten, dass die Kosten einer derartigen elektronisch basierten Vollerhebung deutlich unter den Kosten der bisherigen befragungsbasierten Erhebungen liegen. Zwar könnte die Meldung mit der Erhebung einer Gebühr verbunden werden, um die gesamten Kosten der Erstellung von Mietspiegeln abzudecken. Selbst eine kostendeckende Gebühr würde aber voraussichtlich nur etwa 1 Euro pro Meldevorgang betragen. ${ }^{44}$ Da aktuell die Kosten für die Erstellung des Mietspiegels einer Großstadt in der Regel bereits einen mittleren sechsstelligen Betrag ausmachen, würde die Einrichtung zentraler Datenbanken pro Bundesland jährlich mehrere Millionen an öffentlichen Geldern einsparen.

Eine Möglichkeit, sowohl Missbrauch einzuschränken als auch eine effiziente Kontrolle der Registrierung zu ermöglichen, wäre die Verknüpfung mit der Meldepflicht. Hierzu müsste die ab 15. November 2015 wieder geltende Verpflichtung des Vermieters, den Einzug einer meldepflichtigen Person anzuzeigen ( $\$ 19$ Bundesmeldegesetz), um die Verpflichtung zur Registrierung des Mietvertrag erweitert werden. Dies würde zudem dem Vermieter unnötige

\footnotetext{
${ }^{42}$ So zumindest ein Gutachten im Auftrag von Haus und Grund Deutschland, vgl. Blankennagel et al. (2014, 2015).

${ }^{43}$ Der Verwaltungsaufwand für die Vermieter wäre zwar höher als bisher. Allerdings relativiert sich dieser Aufwand, wenn man voraussetzt, dass sich aufgrund der Mietpreisbremse ohnehin jeder Vermieter vor einer Vermietung über die ortsübliche Vergleichsmiete informieren und hierzu die Daten der zu vermieteten Wohnung erfassen muss. Sofern gesichert ist, dass der Vermieter zur Registrierung auf die bereits eingegeben Daten zurückgreifen kann, relativiert sich der zusätzliche Aufwand erheblich.

${ }^{44}$ Für die Einrichtung einer Datenbank (Programmierung und Infrastruktur) sollten pro Bundesland etwa einmalig 30.000 Euro und ca. 2.000 Euro jährlich anfallen. Hinzu kommen laufende Kosten der Plausibilisierung der Daten und ggf. für das Betreiben eines „Helpdesks“ bei Problemen mit der Bedienung der Datenbank. Hier ist von Personalkosten in Höhe von etwa 100.000 Euro auszugehen. Hinzu kommen die Kosten der Vermieter für die Erfassung der Daten. Nicht berücksichtigt sind ebenfalls Kosten für die Erkennung maschinenlesbarer Formulare oder die Erstellung von Bescheiden an Mieter oder Vermieter. Unter diesen Annahmen wäre eine Gebühr in Höhe von 1 Euro pro Meldevorgang bereits kostendeckend. Die Erhebung der Gebühr wäre dann voraussichtlich teurer als die Gebühr selbst. Bei der Berechnung dieser Kosten wurde von etwa 300.000 Vermietungen pro Bundesland im Jahr ausgegangen. Im Jahr 2014 wurden in Berlin etwa 170.000 Mietverträge neu abgeschlossen und in den zehn größten Städten Bayerns zusammen etwa 140.000 (Schätzung auf Basis der Anzahl der Anzeigen in Immobilienscout24 unter Annahme eines Marktanteils von 80 Prozent in Berlin und 50 Prozent in Bayern, vgl. auch die Schätzung in Börstinghaus (2012), S. 249 f.). Für die Schätzung der EDV-Kosten danken die Autoren Rolf Schillinger, Geschäftsführer FORSEC, Universität Regensburg.
} 
Doppelarbeit ersparen. Auch das Meldeamt würde personell entlastet, da die Informationen nicht schriftlich eingereicht würden, sondern bereits elektronisch vorlägen. ${ }^{45}$

Aus Effizienzgründen und zur Erzielung von Synergieeffekten sollte für die Erhebung, die Plausibilisierung und Verwaltung der Mietdaten auf Verwaltungsstrukturen zurückgegriffen werden, wie sie bei den nach $\S 192 \mathrm{ff}$. BauGB gebildeten amtlichen Gutachterausschüssen bereits vorliegen. Nach § 195 BauGB wird dort eine Vollerhebung aller Verkaufsvorgänge über Immobilien seit 1960 durchgeführt und es liegen umfassende Erfahrungen über den Umgang mit Immobilienmarktdaten vor. Daher bietet es sich an, dass die Mietpreisdatenbanken in die Zuständigkeit der Oberen Gutachterausschüsse bzw. Zentralen Geschäftsstellen fallen, die nach § 198 BauGB in den Ländern eingerichtet sind. ${ }^{46}$ Damit ist eine länderübergreifend einheitlich strukturierte und vergleichbare Bearbeitung der übermittelten Daten vergleichsweise einfach realisierbar. ${ }^{47}$ Datenschutzrechtliche Problematiken sowie Zweifel an der Unabhängigkeit wären mit diesem Ansatz ausgeschlossen.

Zudem ist zu berücksichtigen, dass eine Befragung immer nur eine Stichprobe umfassen kann, deren Qualität und Repräsentativität regelmäßig anfechtbar sind. Durch eine Vollerhebung würden Mietspiegel daher erheblich an Rechtssicherheit gewinnen. Zudem könnte durch die (ggf. auch überregionale) Vollerhebung das Problem der bislang üblicherweise verzerrungsanfälligen Schätzung der ortsüblichen Vergleichsmieten gemindert, evtl. sogar behoben werden. Ein weiterer Vorteil einer fortlaufenden Vollerhebung ist, dass es mit geringem Aufwand möglich wäre, einen Mietspiegel permanent (beispielsweise jährlich oder quartalsweise) fortzuschreiben. Ein weiteres wesentliches Argument für eine Vollerhebung ist, dass die Berechnung des Mietspiegels getrennt von der Erhebung der Daten vergeben werden kann. Da somit der organisatorische Aufwand für den Ersteller des Mietspiegels deutlich geringer ist, eröffnet dies den Marktzugang für eine Vielzahl von weiteren potenziellen Auftragnehmern in Form von Forschungsinstituten und Unternehmen bis hin zu einzelnen Experten, die sich ausschließlich mit der Datenaufbereitung und -auswertung beschäftigen können. Auch sollte ein angemessenes Honorar für die Aufbereitung des Datensatzes und die Berechnung des Mietspiegels nicht mehr als einen kleineren fünfstelligen Betrag betragen, so dass die Kosten des Mietspiegels bei einer Vollerhebung nur einen Bruchteil der bisherigen Kosten umfassen würden.

Schlussendlich muss nicht die Vollerhebung, sondern vielmehr die befragungsbasierte Stichprobenerhebung als unverhältnismäßig aufwändig qualifiziert werden. Dies gilt bereits unter Berücksichtigung des Aufwandes, ganz eindeutig aber bei Abwägung von Aufwand und Nutzen.

\footnotetext{
${ }^{45}$ Dies ist datenschutzrechtlich nicht trivial. Erforderlichenfalls wäre sicherzustellen, dass die Meldedaten und die Vermietungsdaten nur den jeweilig zuständigen Behörden übermittelt werden. Zudem sollte der Mieter über die Daten, die über die von ihm angemieteten Wohnungen erfasst wurden, ebenfalls informiert werden. Zu datenschutzrechtlichen Aspekten bei Mietdatenbanken vgl. Stöwer (2002), S. 68 f., bei der Erstellung von Mietspiegeln vgl. Börtinghaus und Clar (2012), S. 181-189.

${ }^{46}$ Nach Auskunft der Geschäftsstelle des Arbeitskreises der Gutachterausschüsse und Oberen Gutachterausschüsse vom 13.08.2015 sind mit Ausnahme des Landes Baden-Württemberg in allen Ländern Obere Gutachterausschüsse bzw. Zentrale Geschäftsstellen gebildet worden.

47 Da die örtlichen Gutachterausschüsse und sonstige Behörden in die Überprüfung der Daten (Plausibilisierung) eingebunden werden müssen, sollte für umfangreiche Amtshilfe eine entsprechende Kostenverrechnung vereinbart werden.
} 
Empfehlung: Sofern ein Gebiet als angespannter Wohnungsmarkt nach $\S 556 \mathrm{~d}$ Abs. 2 bestimmt wird, sollte verpflichtend ein qualifizierter Mietspiegel nach $\S 558 \mathrm{~d}$ BGB erstellt werden. ${ }^{48}$ Zur Gewährleistung von Repräsentativität sollte das heute gängige Verfahren befragungsbasierter Stichprobenerhebungen durch eine vollständige Erfassung aller Neuvermietungsdaten wenigsten der letzten zwei Jahre in einem elektronischen Register ersetzt werden (Vollerhebung). Für eine effiziente Umsetzung und Kontrolle sollte zum einen die Registrierung der Mietvertragsdaten mit der bereits bestehenden gesetzlichen Verpflichtung des Vermieters zur melderechtlichen Anzeige des Einzugs eines Mieters verbunden werden. Zum anderen sollten die Mietpreisdatenbanken in die Zuständigkeit der Oberen Gutachterausschüsse der Länder fallen.

\subsection{Berücksichtigung besonderer Anbietergruppen sowie Mieter-Vermieter-Verhältnis}

Neben privaten Anbietern, z. B. Privatpersonen oder institutionellen Investoren, sind am Markt auch kommunale Wohnungsbaugesellschaften, Wohnungsbaugenossenschaften, karitative Einrichtungen u. a. aktiv. Diese haben in der Regel den satzungsgemäßen Auftrag, breiten Bevölkerungsgruppen oder ihren Mitgliedern verbilligten Wohnraum zu Verfügung zu stellen. Es ist daher grundsätzlich möglich, dass diese Vermieter Wohnraum systematisch unterhalb der Marktmiete zur Verfügung stellen. Entsprechend sollten die unterschiedlichen Anbietergruppen bei der Berechnung qualifizierter Mietspiegel durch adäquate statistische Methoden berücksichtigt werden, bspw. durch dichotome (kategoriale) Variablen. ${ }^{49}$ Hierdurch ließe sich der oben vermutete Unterschied auch genau messen. Zudem ist die Vorgehensweise unschädlich, sofern sich die Hypothese einer Vermietung unterhalb des Marktniveaus als unzutreffend erweist. ${ }^{50}$ Gleichermaßen sollte bereits bei der Erhebung der Daten festgehalten werden, ob eine Vermietung an nahe Angehörige oder vergleichbar nahestehende Personen erfolgt, um „Gefälligkeitsmieten“ zu identifizieren. $^{51}$

48 So auch Börstinghaus (2014), S. 1-9. Zu den Auswirkungen fehlender qualifizierter Mietspiegel vgl. Abramenko (2015), S. 110f.

49 Des Weiteren muss sichergestellt sein, dass die Gesamtheit nicht nur der befragten, sondern auch aller antwortenden Teilnehmer der Stichprobe repräsentativ für die Gesamtheit aller Mietverhältnisse in dem betroffenen Markt sind. Dies ist bei einer Stichprobe, die keine Vollerhebung ist, grundsätzlich nur durch Erhebung auch von vermieterbezogenen Daten vollends sicherzustellen. Vgl. hierzu Krämer (2014).

50 Eine mögliche und in der Wissenschaft übliche Berücksichtigung dieser Tatsache wäre, diese Anbietergruppen aus der Datenbasis auszuschließen. Die Berücksichtigung der besonderen Eigenschaften dieser Vermietergruppen durch dichotome Variablen in einer Regressionsgleichung hat aber gegenüber einem Ausschluss aus der Datenbasis den Vorteil, dass der postulierte Unterschied in der Miethöhe empirisch überprüft und quantifiziert werden kann. Ein möglicher Nachteil wäre, dass kommunale Wohnungsbaugesellschaften oder Wohnungsbaugenossenschaften Eigenschaften der Wohnung anders als private Vermieter oder gar nicht berücksichtigen, wodurch die Bewertung der spezifischen Ausstattungsmerkmale verzerrt sein könnte. Die adäquate Vorgehensweise ist durch entsprechende Voruntersuchungen zu klären.

${ }^{51}$ Gefälligkeitsmieten werden in der Regel aus dem Datensatz auszuschließen sein, vgl. Börstinghaus und Clar (2013), RN 298. 
Empfehlung: Neben allen wesentlichen wertbestimmenden Merkmalen der Wohnung sollte bei der Datenerhebung erfasst und bei der Auswertung berücksichtigt werden, ob der Vermieter zu einer besonderen Anbietergruppe (kommunale Wohnungsbaugesellschaft, Wohnungsbaugenossenschaft etc.) gehört oder ob die Vermietung an nahe Angehörige erfolgt.

\subsection{Verbesserung des Umgangs mit Unterschieden im Bezugszeitraum der Daten}

Durch die Einbeziehung der Daten der letzten vier Jahre gemäß § 558 Abs. 2 BGB ergibt sich bei einer einfachen Durchschnittsbildung ohne weitere Berücksichtigung des Bezugszeitraums grundsätzlich das Problem, dass Entwicklungen in der Zeit nicht berücksichtigt werden. Dies wäre nur dann korrekt, wenn über die Zeit hinweg keine Strukturveränderungen stattgefunden haben. ${ }^{52}$ Die Bildung eines einfachen Durchschnittes über einen Zeitraum von vier Jahren ohne Berücksichtigung der zeitlichen Unterschiede, wie aktuell üblicherweise bei der Berechnung von Mietspiegeln praktiziert, stellt an sich bereits einen Verstoß gegen anerkannte wissenschaftliche Grundsätze dar, da förmlich „Äpfel mit Birnen“ verglichen werden. Das Problem verstärkt sich, je länger der Erhebungszeitraum ist und wäre bei einem Erhebungszeitraum von zehn Jahren mit wissenschaftlichen Grundsätzen endgültig nicht mehr vereinbar. ${ }^{53}$

Die aktuell praktizierte Methode führt - wiederum unter der Annahme von steigenden Mieten dazu, dass der Mietspiegel im Ergebnis eine ortsübliche Vergleichsmiete ausweist, die unter dem aktuellen Marktniveau liegt. Dies mag politisch gewollt sein. Aus wissenschaftlicher Sicht wäre hingegen zu fordern, dass das Datum der Vereinbarung der Miete im statistischen Verfahren explizit berücksichtigt wird. Bei Regressionsmietspiegeln kann dies durch die Aufnahme einer dichotomen Variablen („Dummy-Variable“) für jeden Zeitraum (z.B. Quartal) in die Regressionsgleichung erfolgen. ${ }^{54}$ Zur Berechnung ortsüblicher Vergleichsmieten ist dann der Dummy für den letzten Erhebungszeitraum auf den Wert eins zu setzen und ermöglicht so eine realitätsnahe und zeitaktuelle Wiedergabe des Mietpreisgefüges auf dem Wohnungsmarkt. ${ }^{55}$

\footnotetext{
${ }^{52}$ Vgl. Maurer et al. (2004), S. 306, Bauer et al. (2013), S.12, Eurostat (2013), S. 51-53.

${ }^{53}$ So dürften im Fall der Vernachlässigung zeitlicher Unterschiede in den Daten bei einer Regression mit hoher Wahrscheinlichkeit autokorrelierte Residuen auftreten. Hypothesentests auf Basis entsprechender Residuen wären statistisch irreführend, vgl. von Auer (2003). S. 410.

${ }^{54}$ Die Regressionsgleichung könnte in Matrixnotation beispielsweise die Form $\mathbf{p}=\mathbf{X} \boldsymbol{\beta}+\boldsymbol{\Omega} \boldsymbol{\gamma} \boldsymbol{\varepsilon}$ haben, wobei $\mathbf{p}=$ Vektor von beobachteten logarithmierten Mietpreisen, $\mathbf{X}=$ Matrix der Eigenschaften der Wohnung mit $\boldsymbol{\beta}$ als Koeffizientenvektor, $\boldsymbol{\Omega}=$ Matrix der Zeitdummys mit $\boldsymbol{\gamma}$ als Koeffizientenvektor und $\boldsymbol{\varepsilon}$ als Vektor der zufallsverteilten Störgrößen. Der Koeffizient $\boldsymbol{\gamma}_{\boldsymbol{i}}$ würde dann angeben, um wieviel Prozent eine Wohnung im Zeitraum $i$ im Durchschnitt teurer oder billiger gegenüber einem Referenzzeitraum wäre. Unter anderem wird hier die zu überprüfende Annahme getroffen, dass die Koeffizienten $\boldsymbol{\beta}$ zeitlich konstant sind. In Abhängigkeit des Datensatzes müssen ggf. alternative Modellierungen getroffen werden, vgl. Maurer et al. (2004), S. 306, Haupt et al. (2010). Im Rahmen des multiplikativ-additiven Mietspiegelmodells („Regensburger Modellansatz“) wären die dichotomen Variablen für den Zeitraum weitere multiplikativ verknüpfte Faktoren, vgl. hierzu Aigner et al. (1993).

55 Eine analoge Umsetzung für Tabellenmietspiegel ist uns nicht bekannt, was die Anwendbarkeit von Tabellenmietspiegeln in diesem Kontext unseres Erachtens weitgehend ausschließt.
} 
Nur unter dieser Voraussetzung können auch Vereinbarungen von Mieten in die Berechnung einbezogen werden, die deutlich (bspw. zehn Jahre) zurückliegen. Durch eine derart ausgeweitete Datenbasis würde sich dann auch die Schätzgenauigkeit deutlich erhöhen. Aus wissenschaftlicher Sicht kann also zur Verbesserung der Schätzgenauigkeit eine Verbreiterung der Datenbasis, z. B. auf die Neuvermietungen der letzten zehn Jahre, sinnvoll sein, sofern der Bezugszeitraum der Daten explizit berücksichtigt wird.

Empfehlung: Die zeitlichen Unterschiede in den Mietpreisdaten sollten explizit bei der Berechnung des qualifizierten Mietspiegels berücksichtigt werden, um statistische Verzerrungen durch unterschiedliche zeitliche Bezugspunkte der Daten auszuschließen. Die Vernachlässigung dieser Unterschiede wird umso bedeutsamer, je länger der Erhebungszeitraum ist.

\subsection{Abbildung der Lage durch räumliches Regressionsmodell}

Bemerkenswert ist, dass das wesentliche Wohnwertmerkmal "Lage“ bei zahlreichen qualifizierten Mietspiegeln gar nicht oder nur unzureichend berücksichtigt wird, obwohl

- der Gesetzgeber dieses Merkmal in § 558 Abs. 2 BGB explizit im Gesetz nennt und

- starke Zusammenhänge zwischen Miethöhe, Lage und weiteren Wohnwertmerkmalen vermutet werden können, so dass bei Nichtberücksichtigung der Lage ein empirisches Modell der Mietenrealität aller Voraussicht nach systematisch verzerrt ist. ${ }^{56}$

Die statistische Berücksichtigung des wesentlichen Wohnwertmerkmals "Lage" wird in der Mietspiegelpraxis bisher sehr uneinheitlich und darüber hinaus kaum nachvollziehbar gehandhabt. Während bei einigen qualifizierten Mietspiegeln lediglich eine sehr grobe Differenzierung in "gute“ und "einfache" Lagen vorgenommen wird, verwenden andere drei oder mehr Lagekategorien. Eine derart grobe Einteilung einer (Groß-)Stadt in einige wenige Wohnlagen wird zwangsläufig nicht werturteilsfrei bzw. objektiv sein. ${ }^{57}$ Bei einem Drittel der qualifizierten Mietspiegel wird die Lage sogar überhaupt nicht berücksichtigt, ${ }^{58}$ wohingegen ausstattungstechnischen Detailfragen oftmals große Aufmerksamkeit zukommt.

Diese Vorgehensweise überrascht, denn die Modellierung von Nachbarschaftseffekten gehört seit Jahrzehnten zum Standardwerkzeugkasten der Ökonometrie ${ }^{59}$ und wird in Handbüchern zur statistischen Analyse von Immobilienpreisdaten dargelegt. ${ }^{60}$ In einem räumlichen Regressionsansatz werden z. B. die durchschnittlichen Mieten geografisch benachbarter Wohnungen explizit als Erklärungsvariable in das Berechnungsmodell aufgenommen. Dem liegt die Annahme zugrunde, dass benachbarte Wohnungen stets einen ähnlichen Wohnlagewert teilen. Der Anteil der durch das Regressionsmodell erklärten Mietenvariation lässt sich damit in aller Regel deutlich verbessern; die

\footnotetext{
${ }^{56}$ Vgl. Promann (2012), S. 111, von Auer (2003), S. 248-250.

${ }^{57}$ Vgl. hierzu umfassend Stelter (2013), Kap. 11.2.3.2.

${ }^{58} \mathrm{Vgl}$. Freund et al. (2013), S. 261.

${ }^{59} \mathrm{Vgl}$. Anselin und Griffith (1988), S. 11-14.

${ }^{60}$ Vgl. z. B. Eurostat (2013), S. 51, Taylor (2008), S. 25.
} 
Einflüsse der anderen Wohnwertmerkmale lassen sich zugleich besser schätzen. ${ }^{61}$ Die statistische Behandlung des sehr vielfältigen und deshalb schwer operationalisierbaren Lagemerkmals wird zugleich objektiviert und erhöht somit die Rechtssicherheit des Mietspiegels. ${ }^{62}$

Die Verarbeitung von Geoinformationen ist mit heutigen Rechnerkapazitäten problemlos möglich und die Informationen sind kostengünstig zu gewinnen. Mithilfe geografischer Informationen ließe sich bspw. eine Karte der Gemeinde erzeugen („Heatmap“), in der die unterschiedlichen Lagen und ihre Mietpreise - bereinigt um übrige Wohnwertmerkmale wie Art, Größe, Ausstattung und Beschaffenheit - anschaulich gemacht werden können. Da zu erwarten ist, dass Wohnungsmärkte über administrative Grenzen hinweg reichen, kann es zur korrekten Berechnung erforderlich sein, auch Daten angrenzender Gemeinden zu berücksichtigen, um den Mietspiegel für eine Gemeinde korrekt zu schätzen. ${ }^{63}$ Vor diesem Hintergrund sollten dem Ersteller des Mietspiegels einer Gemeinde auf Verlangen auch die vollständigen Daten von Gemeinden in unmittelbarer Nachbarschaft zur Verfügung stehen. Zudem sollte sichergestellt sein, dass diese Daten bei der Berechnung von qualifizierten Mietspiegeln auch verwendet werden dürfen.

Die so nach einem objektiv nachvollziehbaren Verfahren gewonnenen Informationen über unterschiedliche Wohnwertlagen in einer Kommune lassen sich nun in unterschiedlicher Weise verwenden. Sinnvollerweise sollte die ortsübliche Vergleichsmiete inkl. der Mietpreisspannen für jede Wohnung individuell durch einen Mietspiegelrechner ermittelt werden, in den auch das räumliche Modell integriert ist. ${ }^{64}$ Die bisherigen Mietspiegel in Papierform würden entfallen.

Zwar wäre es auch mit diesem Verfahren wie bisher möglich, Straßenzüge in ähnlichen Lagen zu gruppieren und diese Gruppierungen dann in Regressionsverfahren, Tabellenmietspiegeln oder hybriden Methoden zu verwenden. Schlussendlich dann könnte ein Mietspiegel wie bisher in Papierform ausgegeben werden. Dies wäre zwar bereits deutlich präziser als die bisherige Praxis, würde jedoch weit hinter den Möglichkeiten des Modells zurückbleiben. Vor allen Dingen ist die Zusammenfassung zu Lagegruppen nicht nur nach objektiven Kriterien möglich und vermindert so wiederum die Rechtssicherheit.

Ein Mietspiegel in Papierform sollte daher nur zu Dokumentationszwecken erstellt werden. Allenfalls hilfsweise sollten diese Mietspiegel bei Vermietungen oder Mieterhöhungen weiterhin als formal

\footnotetext{
${ }^{61}$ Eine Begründung der Vorteilhaftigkeit räumlicher Modelle im Kontext von Mietspiegeln und vergleichbaren Analysen sowie eine verständliche und wenig formale Einführung in die Thematik liefern LeSage und Pace (2009), S. 4-5, 27-28.

62 Freiheitsgrade bestünden überwiegend bei der genauen Berechnungsmethode des Durchschnitts umliegender Wohnungsmieten. Unter Berücksichtigung in der Wirtschaftsgeographie postulierter Wirkungsbeziehungen („First Law of Geography“) würde es sich anbieten, die inverse physische Distanz zu benachbarten Wohnungen als Gewichtung zu verwenden.

${ }^{63} \S 558 c$ Abs. 2 BGB ermöglicht die Erstellung von Mietspiegeln für mehrere Gemeinden. Hier ist aber gemeint, dass für die die Erstellung des Mietspiegels einer Gemeinde auch die Daten anderer Gemeinden verwendet werden.

${ }^{64}$ Siehe dazu auch den „Immobilienpreiskalkulator“ des Oberen Gutachterausschusses für Grundstückswerte in Niedersachsen (www.gag.niedersachsen.de). Hier wird das durchschnittliche Preisniveau für Wohnimmobilien auf Grundlage von Regressionsmodellen bereitgestellt. Die Ergebnisse werden u. a. von der Finanzverwaltung zur Ermittlung von Erbschafts- und Schenkungssteuern genutzt.
} 
zulässige Begründung gelten. Im Konfliktfall sollte aber der Berechnung der ortsüblichen Vergleichsmiete durch einen Mietenrechner höhere Beweiskraft zukommen.

Empfehlung: Die Berücksichtigung der Lage sollte in qualifizierten Mietspiegeln grundsätzlich mittels räumlicher Regressionsmodelle unter Verwendung von Geodatierung erfolgen. Hierzu sollte anstelle eines Mietspiegels in Papierform ein Mietpreisrechner zur Verfügung gestellt werden, der für eine Wohnung die ortsübliche Vergleichsmiete individuell auf Basis des räumlichen Modells berechnet.

\subsection{Verbesserung der beruflichen Qualifizierung}

Vor dem Hintergrund der hohen fachlichen Anforderungen und bedeutsamen finanziellen Auswirkungen für Mieter und Vermieter erscheint es wenig plausibel, dass grundsätzlich jede beliebige Person oder Institution mit der Erstellung eines qualifizierten Mietspiegels beauftragt werden kann. Es sollte daher sichergestellt werden, dass qualifizierten Mietspiegel von solchen Personen oder Stellen erstellt werden, bei denen die Annahme gerechtfertigt ist, dass sie über entsprechende Sachkunde und fachliche Expertise verfügen und in der Lage sind, anerkannte wissenschaftliche Grundsätze umzusetzen. ${ }^{65}$ Hierzu sollte eine berufliche Qualifizierung - z. B. eine bundesweit einheitlich geregelte Zulassungsprüfung zum Mietspiegel-Statistiker/in - geschaffen werden. Die Zulassung zum Mietspiegelstatistiker sollte an das Bestehen einer Prüfung geknüpft sein, in der umfassende Kenntnisse in für Mietspiegel relevanten statistischen Methoden auf dem aktuellen Stand der Forschung nachgewiesen werden müssen. Die Prüfung könnte eine kammerähnliche Institution auf Bundesebene abnehmen. Diese Institution könnte ggf. weitere Standards zur Berufsausübung herausbilden. Zu dieser Prüfung sollten zudem nur Personen zugelassen werden, die über eine adäquate wissenschaftliche Ausbildung verfügen. ${ }^{66}$

Empfehlung: Ein qualifizierter Mietspiegel sollte nur von entsprechend sachverständigen Personen erstellt werden. Hierzu sollte das Berufsbild eines zugelassenen und geprüften MietspiegelStatistikers/-in herausgebildet werden.

\subsection{Stärkung der Dokumentationspflichten}

Aus wissenschaftlicher Sicht sollten die Schritte der Erstellung eines Mietspiegels so dokumentiert und veröffentlicht werden, dass Nachvollziehbarkeit der Ergebnisse durch fremde Dritte möglich ist. Dies umfasst bereits die Vorgehensweise bei der Datenerhebung. Zudem sind alle vorgenommenen Datenaufbereitungen $\mathrm{zu}$ dokumentieren und $\mathrm{zu}$ begründen. Weiterhin ist die konkrete Berechnungsmethode inklusive der Prämissen- und Robustheitstest detailliert zu dokumentieren. Schlussendlich sind die Ergebnisse inklusive der üblichen statistischen Teststatistiken vollständig zu veröffentlichen. Der Zugriff auf die Daten sollte zudem nicht exklusiv auf den Ersteller des

\footnotetext{
${ }^{65}$ Vgl. Aigner et al. (1993), S. 20.

${ }^{66}$ Ein Studium der Statistik wäre sicherlich unstreitig qualifizierend. Denkbar wäre auch ein Studium der Mathematik oder der Wirtschaftswissenschaften mit nachgewiesenem statistischem bzw. ökonometrischem Schwerpunkt.
} 
Mietspiegels beschränkt sein. Stattdessen sollten die Daten bei glaubhaftem Nachweis eines berechtigten Interesses berechtigten Personen für Kontrollrechnungen oder für eigene Zwecke zur Verfügung gestellt werden. Eine einschlägige Tätigkeit als Gutachter, Wissenschaftler oder Sachverständiger bzw. die Zulassung als Mietspiegel-Statistiker sollte dabei grundsätzlich ein berechtigtes Interesse begründen. ${ }^{67}$

Empfehlung: Die Vorgehensweise bei der Datenerhebung, Datenbearbeitungen, die konkrete Berechnungsmethode sowie die Ergebnisse selbst sollten vollständig und so detailliert zu veröffentlichen sein, dass eine Replikation der Berechnungen möglich ist. Weiterhin sollte gewährleistet sein, dass auch die Daten selbst für Überprüfungen oder andere wissenschaftliche oder statistische Zwecke zur Verfügung stehen.

\subsection{Unabhängigkeit von politischer Einflussnahme}

In einem Rechtsstaat werden amtliche Statistiken nicht ohne Grund von einer unabhängigen Behörde erstellt. Im Gegensatz dazu unterliegt der Mietspiegel trotz seiner hohen Bedeutung zu einem großen Teil dem Gestaltungswillen lokaler Politiker und Interessensvertreter. Es muss sichergestellt sein, dass der Mietspiegel tatsächlich seine primäre Funktion als Spiegel der Mietverhältnisse auf dem jeweiligen Wohnungsmarkt erfüllt und nicht etwa das Ergebnis politischer oder interessenspolitischer Einflussnahme darstellt. Zunächst sollte daher die gesetzliche Erfordernis der Zustimmung von Gemeinde oder Interessenvertretern der Vermieter und der Mieter gemäß §558d Abs. 1 BGB entfallen. Die einfachste und zugleich sicherste Möglichkeit, um Unabhängigkeit zu gewährleisten, wäre eine Übertragung der Zuständigkeit nicht nur für die Datenerhebung, sondern auch für die Ausschreibung der Berechnung der Mietspiegel auf die Oberen Gutachterausschüsse. Diese Gutachterausschüsse sind entsprechend ihres gesetzlichen Auftrags bereits zur Unabhängigkeit verpflichtet (§ 198 Abs. 1 Satz 2 i. V. m. § 192 Abs. 1 BauGB).

Sofern aus politischen Gründen eine Begrenzung des Mietwachstums erreicht werden soll, muss dies dann getrennt von der Berechnung des Mietspiegels umgesetzt werden. Die Mietpreisbremse kann hier als Vorbild dienen: bei Wiedervermietungen nicht mehr als zehn Prozent über der ortsüblichen Vergleichsmiete. Eine entsprechende Regelung für Mieterhöhungen würde dann noch fehlen. Ob hier ein Abschlag von null, zehn oder dreißig Prozent zur ortsüblichen Vergleichsmiete umgesetzt wird, bleibt allein der Politik vorbehalten.

Mietpreiskontrollen, die dauerhaft marktgerechten Mieten verhindern, sind aus ökonomischer Sicht weiterhin abzulehnen. Dennoch wäre die oben skizzierte Regelung bereits deutlich transparenter und präziser als die bisherige Praxis der politischen und interessenspolitischen Einflussnahme auf die Mietspiegelerstellung. Nicht zuletzt könnte hierdurch eine deutliche Steigerung der Rechtssicherheit erreicht werden.

Empfehlung: Die Zuständigkeit für Ausschreibung und Vergabe der Erstellung qualifizierter Mietspiegel sollten bei den Oberen

\footnotetext{
${ }^{67}$ Dies ist aus datenschutzrechtlicher Sicht nicht trivial. Hier ist eine geeignete Abwägung zwischen Anonymisierung und Aussagekraft der Daten zu treffen.
} 
Gutachterausschüssen der Länder liegen, um die politische und interessenspolitische Unabhängigkeit zu gewährleisten.

Eine politisch motivierte Begrenzung des Mietwachstums sollte getrennt von der Berechnung des Mietspiegels umgesetzt werden. Hierzu könnte eine Begrenzung der Mieterhöhungen nach dem Vorbild der Mietpreisbremse ergänzt werden. Beispielsweise könnten Mieterhöhungen nur bis zu einem festzulegenden Prozentsatz unterhalb der (marktgerechten) ortsüblichen Vergleichsmiete möglich sein.

\subsection{Keine Detailvorgaben zu Datenaufbereitung und Modellspezifikation}

In den vorstehenden Abschnitten wurden zu wesentlichen organisatorischen und inhaltlichen Punkten ambitionierte, jedoch aus unserer Sicht praktikable Vorschläge gemacht, die dazu beitragen können, die Regulierung des (qualifizierten) Mietspiegels vor dem Hintergrund der Mietpreisbremse zu verbessern. Es stellt sich die Frage, ob darüber hinaus noch weitere Vorgaben durch den Gesetzgeber sinnvoll sind. Möglich wären hier etwa konkrete inhaltliche Vorgaben zur Berechnungsmethode, zur Operationalisierung der im Rahmen der Datenerhebung verpflichtend zu berücksichtigenden wohnwertbestimmenden Merkmale der "Ausstattung“ bzw. „Beschaffenheit", zur Extremwertbereinigung, zur Ausgabe von Mietpreisspannen etc.

Bereits die Vorgabe der relevanten wohnwertbestimmenden Daten ist nicht trivial, da streng genommen alle relevanten Merkmale erfasst werden müssen, diese ex ante aber nicht bekannt sind und zudem lokal erheblich unterschiedliche Bedeutung haben können. Sinnvollerweise wird man im Zweifelsfall versuchen, möglichst viele Merkmale zu erheben. Es wird in der Praxis aber regelmäßig nicht umsetzbar sein, sämtliche denkbaren erklärenden Merkmale zu berücksichtigen.

Werden anschließend zur Identifikation bzw. Quantifizierung des Einflusses der Merkmale Regressionen verwendet, stellt sich zudem die Frage der konkreten Modellspezifikation. Hier wäre etwa zu überprüfen, ob die Voraussetzungen zur Anwendung einer linearen Regression erfüllt sind oder ob nichtlineare Regressionsmodelle verwendet werden müssen. ${ }^{68}$ Weiterhin wäre zu klären, welche Variablen in welcher Kombination zur Erklärung der Mieten herangezogen werden sollen oder in welcher Form Interaktionen zwischen den Variablen zu berücksichtigen sind. Zudem wäre festzulegen, ob und ggf. in welcher Form eine Extremwertbereinigung durchzuführen ist. Schlussendlich wäre nach korrekter Berechnung auch noch zu klären, in welcher Höhe Mietpreisspannen auszugeben sind.

Zu diesen Fragen gibt es keine einvernehmliche Antwort und insbesondere keine, die für alle Wohnungsmärkte gültig wäre. Eine präzise Vorgabe durch den Gesetzgeber, der die lokalen Gegebenheiten und (selbst nur die wesentlichen) statistischen Probleme berücksichtigt, ist daher weder sinnvoll noch möglich. Wichtig ist jedoch, dass die Entscheidungen grundsätzlich nach nachvollziehbaren statistischen bzw. wissenschaftlichen Prinzipien getroffen werden und nicht als Verhandlungsergebnis zwischen Kommunalpolitikern und den Interessensvertretungen von Mietern

${ }^{68}$ Vgl. zur Wahl der korrekten Funktionsform Maurer et al. (2004), S. 315-316. Zur Zulässigkeit von Regressionsmietspiegeln oder Tabellenmietspiegeln vgl. Krämer 1992, Klein und Martin (1994), S. 144 ff., Keller (1996), S. 111 ff. sowie Börstinghaus und Börstinghaus (2003), S. 381 f. 
und Vermietern, welches sich einer objektiven Überprüfung entzieht. Ein derartiger Kompromiss wird auch nur im Ausnahmefall einer wissenschaftlichen Überprüfung standhalten und wäre damit auch rechtlich angreifbar. In der Praxis kann nur - wie in der Forschung üblich - der wissenschaftliche Wettbewerb über die tauglichsten Modelle entscheiden. ${ }^{69}$

Für weitere Detailfragen hat der Gesetzgeber mit dem Kriterium der anerkannten wissenschaftlichen Grundsätze bereits genau die Vorgabe gegeben, die es ermöglicht, dass sich Mietspiegel dem Stand der Forschung und Rechtsprechung anpassen können und müssen. Beispielsweise wird zu Extremwerten in den „Hinweisen zur Erstellung von Mietspiegeln“ des BMVBS zutreffend ausgeführt: „Beim qualifizierten Mietspiegel ist auf eine statistisch fundierte Eliminierung von Ausreißern zu achten. Die Eliminierung darf nicht auf der Basis willkürlicher Festlegungen, z. B. durch den Arbeitskreis Mietspiegel, erfolgen. ${ }^{\text {"70 }}$ In der bisherigen Praxis der Erstellung von Mietspiegeln wird die „Extremwertbereinigung" bislang häufig als simples Verkürzen der Verteilung vor Durchführen der Regression praktiziert, was wissenschaftlichen Grundsätzen nur bei Vorliegen von Messfehlern gerecht wird (beispielsweise einer Wohnfläche von $1 \mathrm{~m}^{2}$ ). ${ }^{71}$ Insofern sollten auch bislang allgemein übliche Vorgehensweisen kritisch überprüft werden, ob diese mit wissenschaftlichen Grundsätzen vereinbar sind. ${ }^{72}$

Auch die Festlegung der Mietpreisspannen ist nicht wissenschaftlich begründet, sondern hat sich als allgemeine Praxis etabliert. Hierzu ist ebenso festzustellen, dass eine allgemeine Praxis weder richtig noch rechtssicher sein muss. ${ }^{73}$ Ebenso wenig garantiert sie eine Übereinstimmung mit wissenschaftlichen Grundsätzen. ${ }^{74}$ So werden Mietpreisspannen, insbesondere bei Tabellenmietspiegeln, häufig als „2/3-Spannen“ berechnet. ${ }^{75}$ Hiermit ist gemeint, dass zwei Drittel der für die Betrachtung relevanten Mietwerte innerhalb der Spanne liegen, und zwar mit dem Ziel, eine „einvernehmliche Beurteilung der Beteiligten ${ }^{476} \mathrm{zu}$ erreichen. Eine abstrakte Diskussion über konkrete Prognoseintervalle bzw. Mietpreisspannen ist losgelöst vom tatsächlichen Modell und Datensatz kaum möglich. ${ }^{77}$ Zudem entzieht sich die Festlegung von Mietspannen bzw. Prognoseintervallen als

\footnotetext{
${ }^{69}$ Vgl. von Auer (2003), S. 267.

${ }^{70} \mathrm{Vgl}$. Bundesministerium für Verkehr, Bau- und Wohnungswesen (2002), S. 28.

${ }^{71}$ Auch Mietpreise, die bei einer univariate Analyse der Mietenverteilung zunächst als Ausreißer erscheinen, können sich nach Kontrolle erklärender Wohnwertmerkmale durchaus als „normal“ erweisen. Beispielsweise wird ein sehr hoher Mietpreis beobachtet, dieser erklärt sich jedoch im Rahmen der Regression durch eine sehr gute Lage und Ausstattung etc. Eine Herausforderung stellen dagegen Fälle mit ungewöhnlichen Merkmalskombinationen (multivariate Ausreißer) dar, wie etwa ein sehr niedriger Mietpreis für eine Wohnung mit ebenfalls sehr guter Ausstattung in sehr guter Lage. Diese Fälle können die Regressionsergebnisse maßgeblich beeinflussen, sind jedoch nicht durch reine Betrachtung der Mietenverteilung feststellbar. Entsprechende Fälle sind anhand geeigneter Verfahren im Zuge der Regressionsanalyse selbst zu identifizieren. Vgl. hierzu auch zum Mietspiegels Berlin durch Krämer (2014), S. 8-9. Für eine kritische Würdigung von Krämer (2014) siehe Börstinghaus (2015).

${ }^{72}$ Vgl. Stelter (2015).

${ }^{73}$ Vgl. Börstinghaus und Clar (2013), RN 302.

${ }^{74}$ Zum Begriff der Üblichkeit und zu Mietpreisspannen aus rechtlicher Sicht vgl. Börstinghaus, U. (2012).

${ }^{75}$ Vgl. Bundesministerium für Verkehr, Bau- und Wohnungswesen (2002), S. 41-42.

${ }^{76} \mathrm{Vgl}$. Bundesministerium für Verkehr, Bau- und Wohnungswesen (2002), S. 41.

${ }^{77}$ In empirischen Untersuchungen wird üblicherweise mit Konfidenz- oder Prognoseintervallen von 90 Prozent und mehr gearbeitet. Das ungewöhnlich niedrige Prognoseintervall von 67 Prozent bei Mietspannen ist die Konsequenz der häufig vergleichsweise geringen statistischen Erklärungskraft der Mietspiegelmodelle. Bei
} 
normativer Wert weitgehend statistischen Erwägungen. ${ }^{78}$ Sinnvollerweise sollte aber auch die Festlegung der Breite einer Mietpreisspanne nicht zum Gegenstand politischer Verhandlung werden, sondern entweder vom Ersteller des Mietspiegels oder von den Oberen Gutachterausschüssen als neutrale Instanz festgelegt werden.

Ein Methodenstreit mit der Folge, dass im Ergebnis weniger statt mehr Rechtssicherheit herrscht, ist bei Ermangelung konkreter inhaltlicher Gesetzesvorgaben zu Datenaufbereitung und Modellspezifikation nicht zu befürchten. Erst wenn eine andere Vorgehensweise nachweist, dass bei der Berechnung eines Mietspiegels z. B. relevante wertbegründende Faktoren ignoriert oder hinlänglich bekannte wissenschaftliche Techniken außer Acht gelassen wurden und dies zu wesentlichen Abweichungen geführt hat, kann ein Verstoß gegen anerkannte wissenschaftliche Grundsätze bestehen. Die gesetzliche Anforderung, dass anerkannte wissenschaftliche Grundsätze erfüllt sein sollen, bedeutet eben gerade nicht, dass nur eine Form der Berechnung eines Mietspiegels "richtig“ ist. Im wissenschaftlichen Diskurs wird es in Detailfragen in der Regel nicht nur eine, sondern mehrere unterschiedliche Meinungen geben, die anerkannte wissenschaftliche Grundsätze erfüllen. Ein Beispiel wäre etwa, ob und ggf. mit welcher Methode mögliche Interaktionen zwischen verschiedenen wohnwertbestimmenden Merkmalen (z. B. Wohnfläche und Einbauküche) im Modell berücksichtigt werden. Zudem unterliegen statistische Methoden ebenso wie die angewandte empirische Forschung immer einer Weiterentwicklung. Sofern ein anderer Ansatz zu besseren Ergebnissen kommt, handelt es sich daher zunächst nur um eine Weiterentwicklung bzw. Verbesserung des ursprünglichen Ansatzes. Ein Mietspiegel wäre daher grundsätzlich auch dann noch nach anerkannten wissenschaftlichen Grundsätzen erstellt - und damit rechtssicher -, wenn eine andere Berechnungsmethode zu abweichenden, aber qualitativ vergleichbaren Ergebnissen kommen würde.

Empfehlung: Detaillierte gesetzliche Vorgaben zu Datenaufbereitung und Modellspezifikation sind nicht sinnvoll. Die gesetzliche Verpflichtung der Einhaltung wissenschaftlicher Grundsätze, die fachliche Qualifikation des Erstellers sowie die Unabhängigkeit der beauftragenden Institution sind ausreichende Anforderungen.

\footnotetext{
Intervallen von 90 Prozent und mehr würden die Mietspannen vieler Mietspiegel aussagelos werden, da dann nahezu jede Miethöhe „üblich“ wäre. Wir geben dennoch zu bedenken, dass bei einem derart großzügigen Intervall in Kauf genommen wird, dass Mietpreise mit einer Wahrscheinlichkeit von 33 Prozent als nicht „üblich“ klassifiziert werden. Es bedeutet auch, dass ein Drittel der am Markt tatsächlich beobachteten Mieten als „zu hoch“ "bzw. „zu niedrig“ interpretiert werden. Unter den Voraussetzungen einer Vollerhebung in der von uns vorgeschlagenen Form ist zu erwarten, dass sich die Verlässlichkeit der Berechnung der ortsüblichen Miete anhand von Regressionsmietspiegeln deutlich verbessert wird. Zusätzlich zur mittleren Prognose für die ortsübliche Miete ließen sich anhand von Regressionsmietspiegeln auch empirisch fundierte, exaktere Vertrauensintervalle der üblichen Miete berechnen. Mietpreisspannen von 2/3 werden dann hoffentlich nicht mehr notwendig sein.

${ }^{78}$ Vgl. Oberhofer/Schmidt (2014), S. 30-34.
} 


\section{Fazit}

In der wissenschaftlichen Literatur werden Preiskontrollen auf Wohnungsmärkten kritisiert, da diese gemäß empirischer Evidenz in der Regel einen Rückgang des Angebots an Mietwohnungen und negative Markteffekte zu Lasten vor allem der sozial schwachen Mieter hatten. Der Gesetzgeber hat sich dennoch entschieden, die Höhe der Mieten durch die sogenannte Mietpreisbremse zu begrenzen. Trotz aller Kritik ist der Mietpreisbremse zu attestieren, dass sie den politischen Gestaltungswillen in transparenter Form zum Ausdruck bringt: Bei Neuvermietungen darf die Miete die ortsübliche Vergleichsmiete nur um maximal zehn Prozent übersteigen. Allerdings ist die Erstellung qualifizierter Mietspiegel bislang weitgehend intransparent und in substantiellem Ausmaß von politischer Einflussnahme betroffen. Im Ergebnis ist die ortsübliche Vergleichsmiete gegenüber der Marktrealität durch politische Einflussnahme systematisch nach unten verzerrt. In Kombination mit der Mietpreisbremse impliziert dies eine faktische Preisabsenkung, die ökonomisch einer Sondersteuer auf Mieteinnahmen gleichkommt.

Ein qualifizierter Mietspiegel sollte die tatsächlichen Verhältnisse auf den Wohnungsmärkten im Sinne einer Marktorientierung realitätsnah widerspiegeln. Zum einen kann so der durch die Mietpreisbremse gewünschte Effekt erzielt werden, dass bei vorübergehender Marktknappheit die Mieten nicht übermäßig steigen. Trotzdem wäre durch eine korrekte Abbildung des Marktpreisniveaus in der ortsüblichen Vergleichsmiete gewährleistet, dass die Mieten - wenngleich mit Verzögerung - das volkswirtschaftlich richtige Preissignal setzen, um weiterhin eine Versorgung der Wohnungssuchenden mit privatem Wohnraum zu gewährleisten. Aus unserer Sicht sollte daher der mit der Mietpreisbreme eingeschlagene Weg insofern weiter verfolgt werden, als dass Statistik und politisch gewollte Mietpreiskontrolle klar getrennt werden, wie in einem Rechtsstaat üblich. Sofern eine Begrenzung der Miethöhe beabsichtigt ist, sollte dies in transparenter Form getrennt von der Berechnung des Mietspiegels gesetzlich geregelt werden. Die Mietpreisbremse kann hierbei als Vorbild für eine mögliche analoge Regelung für Mieterhöhungen in bestehenden Mietverhältnissen dienen.

Eine hinreichend präzise Schätzung der ortsüblichen Vergleichsmiete ist nur möglich, wenn hierzu die entsprechenden rechtlichen und organisatorischen Voraussetzungen geschaffen werden. Von entscheidender Bedeutung ist hierbei zum einen die Datenerhebung. Aus Gründen der Kosteneffizienz, einer Reihe von Vorteilen aus statistischer Sicht sowie der Rechtssicherheit schlagen wir eine internetbasierte Vollerhebung aller Neuvermietungen vor. Auf die Erfassung von Mieterhöhungen und Staffelmieten kann verzichtet werden, da diese keinen Bezug zum aktuellen Marktgeschehen aufweisen.

Aus wissenschaftlicher Sicht ist weiterhin die Verbesserung der Berechnungspraxis qualifizierter Mietspiegel geboten. Dies betrifft sowohl die Berücksichtigung besonderer Anbietergruppen als auch den Umgang mit Daten aus unterschiedlichen Erhebungszeiträumen. Auch die Berücksichtigung der Lage als eines der wichtigsten wertbestimmenden Kriterien einer Wohnung sollte dem Stand der Forschung angepasst werden. Weiterhin sollte die nach wissenschaftlichen Grundsätzen gebotene Transparenz umfassend eingehalten werden. So sollten die Angaben zur Datenerhebung, Datenaufarbeitung und Datenverarbeitung in einer Form dargelegt werden, die eine Neuberechnung zur Kontrolle ermöglichen.

Im Übermaß detaillierte Vorgaben für die Berechnung von Mietspiegeln halten wir hingegen nicht für sinnvoll, da die spezifischen Charakteristika des individuellen Datensatzes nicht abschließend 
berücksichtigt werden können. Stattdessen schlagen wir zum einen vor, strenge Anforderungen an die Qualifikation der Ersteller von Mietspiegeln zu schaffen und das Berufsbild eines/r MietspiegelStatistikers/in zu etablieren. Zum anderen halten wir es zusätzlich für erforderlich, dass eine unabhängige Instanz mit Datenerhebung und Vergabe der Berechnung beauftragt wird, um die politische Unabhängigkeit des qualifizierten Mietspiegels zu garantieren. Hier bieten sich aufgrund der einschlägigen Sachkenntnis und der gesetzlich garantierten Unabhängigkeit insbesondere die Oberen Gutachterausschüsse der Länder an. Diese sind derzeit bereits mit der Vollerhebung und Auswertung aller Verkaufsvorgänge auf dem Immobilienmarkt befasst.

In Summe sollte durch unsere Vorschläge erreichbar sein, dass die Qualität, die Akzeptanz und letztlich auch die Rechtssicherheit qualifizierter Mietspiegel deutlich erhöht werden kann. 


\section{Literaturverzeichnis}

Abramenko, A. (2015): Die Mietpreisbremse, Bonn.

Aigner K., W. Oberhofer. und B. Schmidt (1993): Eine neue Methode zur Erstellung eines Mietspiegels am Beispiel der Stadt Regensburg, Wohnungswirtschaft und Mietrecht (WM) 46, S. 16-21.

Andrews, D., A. Caldera-Sánchez und A. Johansson (2011): Housing Markets and Structural Policies in OECD Countries, Organisation for Economic Co-operation and Development (OECD) ECO/WKP(2011) 5, Paris.

Anselin, L. und D. A. Griffith (1988): Do spatial effects really matter in regression analysis? Papers in Regional Science 65 (1), S. 11-34.

Auer, L. von (2003): Ökonometrie - Eine Einführung, Köln.

Bauer, T. K., S. Feuerschütte, M. Kiefer, P. an de Meulen, M. Micheli, T. Schmidt und L.-H. Wilke (2013): Ein hedonischer Immobilienpreisindex auf Basis von Internetdaten: 2007-2011, AStA Wirtschafts- und Sozialstatistisches Archiv 7 (1-2), S. 5-30.

Blankenagel, A., R. Schröder und W. Spoerr (2014): Gutachten - Verfassungsmäßigkeit des Instituts und der Ausgestaltung der sog. Mietpreisbremse auf Grundlage des Gesetzesentwurfs zur Dämpfung des Mietanstiegs auf angespannten Wohnungsmärkten und zur Stärkung des Bestellerprinzips bei der Wohnungsvermittlung (Mietrechtsnovellierungsgesetz - MietNovG), mimeo.

Blankenagel, A., R. Schröder und W. Spoerr (2015): Verfassungsmäßigkeit des Instituts und der Ausgestaltung der sog. Mietpreisbremse auf Grundlage des MietNovGE, Neue Zeitschrift für Mietund Wohnungsrecht (NZM) 18, S. 1-28.

Blinkert, B. und P. Höfflin (1994): Die Qualität von Mietspiegeln als Modelle des Wohnungsmarktes Tabelle oder Regression? Ein empirischer Beitrag zur Methodendebatte; Wohnungswirtschaft und Mietrecht (WM) 47, S. 589-595.

Börstinghaus, U. (2012): Der Begriff der ortsüblichen Vergleichsmiete und wie deren Höhe richtig zu ermitteln ist - Über Spannen, Bandbreiten, Ausreißer und Üblichkeit - eine Besprechung von BGH, Urteil vom 29. Februar 2012 - VIII ZR 346/10, Wohnungswirtschaft und Mietrecht (WM) 65, S. 244252.

Börstinghaus, U. (2014): Stellungnahme aus amtsrichterlicher Sicht zum mietrechtlichen Teil des Entwurfs eines Gesetzes zur Dämpfung des Mietanstiegs auf angespannten Wohnungsmärkten und zur Stärkung des Bestellerprinzips bei der Wohnungsvermittlung (Mietrechtsnovellierungsgesetz MietNovG) BT-Drs 18/3121, download.

Börstinghaus, U. (2015): Berliner Mietspiegel zwischen Qualifikation und Disqualifikation Anmerkung zu AG Berlin-Charlottenburg, Urteil vom 27.02.2015, 232 C 262/14, Miet- und Wohnungseigentumsrecht (jurisPR-MietR), Anm. 1.

Börstinghaus, U. und C. Börstinghaus (2003): Qualifizierte Mietspiegel in der Praxis - eine empirische Untersuchung über Akzeptanz und praktische Umsetzung des neuen Rechtsinstruments, Neue Zeitschrift für Miet- und Wohnungsrecht (NZM) 6, S. 377-386.

Börstinghaus, U. und M. Clar (2013): Mietspiegel - Probleme der Erstellung und Anwendung von Mietspiegeln aus juristischer und statistischer Sicht, 2. Auflage, München. 
Börstinghaus, U. und M. Clar (2014): Die Ermittlung der ortsüblichen Vergleichsmiete, Vortrag auf dem 16. Deutschen Mietgerichtstag, 4.-5. April 2014, Dortmund, download, veröffentlicht in: Neue Zeitschrift für Miet- und Wohnungsrecht (NZM) 17, S. 889-901.

Bundesministerium für Verkehr, Bau- und Wohnungswesen (2002): Hinweise zur Erstellung von Mietspiegeln, Berlin.

Bundesministerium für Verkehr, Bau- und Wohnungswesen (2013): Umwandlungsprojekte von Nichtwohngebäuden in Studentenwohnungen. BMVBS-Online-Publikation 10/2013, download.

Bundesregierung (2013): Deutschlands Zukunft gestalten. Koalitionsvertrag zwischen CDU, CSU und SPD, 18. Legislaturperiode, Berlin.

Deschermeier, P., H. Haas, M. Hude und M. Voigtländer (2014): Die Folgen der Mietpreisbremse Eine Analyse am Beispiel der Wohnungsmärkte in Köln und Berlin, IW Policy Paper 17/2014.

Deutsche Forschungsgemeinschaft (2013): Vorschläge zur Sicherung guter wissenschaftlicher Praxis, 2013, ergänzte Auflage, download.

Eekhoff, J. (2002): Wohnungspolitik, Tübingen.

Eucken, W. (1952): Grundsätze der Wirtschaftspolitik, Tübingen.

Eurostat (2013): Handbook on Residential Property Prices Indices (RPPIs), Luxembourg.

Freund, S., V. Hilla, D. Missal, J. Promann und B. Woeckener (2013): Qualifizierte Mietspiegel: Verbreitung, Standardisierungsmöglichkeiten und Qualitätsdefizite, Wohnungswirtschaft und Mietrecht (WM) 66, S. 259-263.

Fritsch, T. Wein und H.-J. Ewers (2006): Marktversagen und Wirtschaftspolitik: Mikroökonomische Grundlagen staatlichen Handelns, München.

Glaeser, E. L. und E. F. P. Luttmer (2003): The Misallocation of Housing under Rent Control, American Economic Review 93 (4), S. 1027-1046.

Haupt, H., J. Schnurbus und R. Tschernig (2010): On Nonparametric Estimation of a Hedonic Price Function, Journal of Applied Econometrics 25 (5), S. 894-901.

Held, T., J. Nielsen, A. Schürt und M. Waltersbacher (o. J.): Hintergrundpapier: Aktuelle Mietenentwicklung und ortsübliche Vergleichsmiete: Liegen die erzielbaren Mietpreise mittlerweile deutlich über dem örtlichen Bestandsmietenniveau?, download.

Klein, T. und F. O. Martin (1994): Tabellenmethode versus Regressionsmethode bei der Erstellung von Mietspiegel. Ein empirischer Vergleich, Wohnungswirtschaft und Mietrecht (WM) 47, S. 144-150.

Krämer, W. (1992): Pro und Contra die Erstellung von Mietspiegeln mittels Regressionsanalyse, Wohnungswirtschaft und Mietrecht (WM) 45, S. 172-175.

Krämer, W. (2014): Gutachterliche Stellungnahme zu den statistischen Aspekten des Berliner Mietspiegels 2013, insbesondere zu den Fragen, ob die Wohnlageklassifizierung und die Abhängigkeit der ortsüblichen Vergleichsmiete von Sondermerkmalen anerkannten wissenschaftlichen Standards entspricht, mimeo.

Lerbs, O. (2014): Germany's rental property sector: A cornerstone of housing market resilience?, Housing Finance International 2, S. 38-44.

LeSage, J. und K. R. Pace (2009): Introduction to Spatial Econometrics, Boca Raton. 
Maurer, R., M. Pitzer, S. P. Sebastian (2004): Hedonic Price Indices for the Paris Housing Market. Journal of the German Statistical Society (Allgemeines Statistisches Archiv) 88, S. 1-24.

Niederberger, R. (1980): Mietspiegel als Instrument zur Ermittlung der ortsüblichen Vergleichsmiete, Köln.

Oberhofer, W. und B. Schmidt (2014): Der Regensburger Mietspiegel 2014 - Arbeitsbericht.

Olsen, E. O. (1991): Is Rent Control Good Social Policy?, Chicago-Kent Law Review 67 (3), S. 931-945.

Patrizia Immobilien AG (2014): Europäische Wohnungsmärkte 2014/2015, download.

Promann, J. (2012): Die Berücksichtigung des Wohnwertmerkmals Lage in den Mietspiegeln deutscher Großstädte, Dissertation, Universität Stuttgart.

Richter, R. und E. G. Furubotn (2003): Neue Institutionenökonomik, Tübingen.

Sachverständigenrat zur Begutachtung der gesamtwirtschaftlichen Entwicklung, Hrsg. (2013): Gegen eine rückwärtsgewandte Wirtschaftspolitik - Jahresgutachten 2013/14.

Schlittgen, R. und S. Uhlig (1997): Repräsentativität von Mietspiegeln, Wohnungswirtschaft und Mietrecht (WM) 50, S. 314-316.

Sims, D. P. (2007): Out of control: What can we learn from the end of Massachusetts rent control? Journal of Urban Economics 61 (1), S. 129-151.

Skak, M. und G. Bloze (2013): Rent Control and Misallocation, Urban Studies 50 (10), S. 1-18.

Stelter, M. (2013): Mietpreisbewertung, in: Gerady, T., R. Möckel, H. Troff, B. Bischoff (Hrsg.): Praxis der Grundstücksbewertung (Stelter), 104. Ausgabe 12/2013, München.

Stelter, M. (2015): Mietspiegel und Mietgutachten - Probleme aus Sicht eines Mietensachverständigen, erscheint in: Das Grundeigentum, September 2015.

Stöver, B. (2002): Mietdatenbanken nach neuem Recht, Wohnungswirtschaft und Mietrecht (WM) 55, S. $65-70$

Taylor, L. O. (2008): Theoretical Foundations and Empirical Developments in Hedonic Modeling, in: Baranzini, A., Ramirez, J., Schaerer, C. und P. Thalmann (Hrsg.): Hedonic Methods in Housing Markets, New York. 